\title{
Isoform-Selective Susceptibility of DISC1/ Phosphodiesterase-4 Complexes to Dissociation by Elevated Intracellular cAMP Levels
}

\author{
Hannah Murdoch, ${ }^{1 \star}$ Shaun Mackie, ${ }^{2 \star}$ Daniel M. Collins, ${ }^{1}$ Elaine V. Hill, ${ }^{1}$ Graeme B. Bolger, ${ }^{4}$ Enno Klussmann, ${ }^{3}$ \\ David J. Porteous, ${ }^{2}$ J. Kirsty Millar, ${ }^{2}$ and Miles D. Houslay ${ }^{1}$ \\ ${ }^{1}$ Molecular Pharmacology Group, Division of Biochemistry and Molecular Biology, Institute of Biomedical and Life Sciences, University of Glasgow, \\ Glasgow G12 8QQ, United Kingdom, ${ }^{2}$ Medical Genetics Section, Molecular Medicine Centre, University of Edinburgh, Edinburgh EH4 2XU, United \\ Kingdom, ${ }^{3}$ Leibniz-Institut für Molekulare Pharmakologie, Campus Berlin-Buch, 13125 Berlin, Germany, and ${ }^{4}$ Comprehensive Cancer Center, University of \\ Alabama at Birmingham, Birmingham, Alabama 35294-3300
}

\begin{abstract}
Disrupted-in-schizophrenia 1 (DISC1) is a genetic susceptibility factor for schizophrenia and related severe psychiatric conditions. DISC1 is a multifunctional scaffold protein that is able to interact with several proteins, including the independently identified schizophrenia risk factor phosphodiesterase-4B (PDE4B). Here we report that the $100 \mathrm{kDa}$ full-length DISC1 isoform (fl-DISC1) can bind members of each of the four gene, cAMP-specific PDE4 family. Elevation of intracellular cAMP levels, so as to activate protein kinase A, caused the release of PDE4D3 and PDE4C2 isoforms from fl-DISC1 while not affecting binding of PDE4B1 and PDE4A5 isoforms. Using a peptide array strategy, we show that PDE4D3 binds fl-DISC1 through two regions found in common with PDE4B isoforms, the interaction of which is supplemented because of the presence of additional PDE4B-specific binding sites. We propose that the additional binding sites found in PDE4B1 underpin its resistance to release during cAMP elevation. We identify, for the first time, a functional distinction between the $100 \mathrm{kDa}$ long DISC1 isoform and the short $71 \mathrm{kDa}$ isoform. Thus, changes in the expression pattern of DISC1 and PDE4 isoforms offers a means to reprogram their interaction and to determine whether the PDE4 sequestered by DISC1 is released after cAMP elevation. The PDE4B-specific binding sites encompass point mutations in mouse Discl that confer phenotypes related to schizophrenia and depression and that affect binding to PDE4B. Thus, genetic variation in DISC1 and PDE4 that influence either isoform expression or docking site functioning may directly affect psychopathology.
\end{abstract}

Key words: cAMP; phosphodiesterase-4; PDE4; DISC1; rolipram; scaffold proteins

\section{Introduction}

The discovery of a balanced chromosomal translocation cosegregating with major psychiatric disorders identified a lesion within the open reading frame (ORF) of disrupted-in-schizophrenia 1 (DISC1), leading to the DISC1 locus being identified as a risk factor for schizophrenia and other mental illnesses (Porteous et al., 2006). DISC1 has now been demonstrated to influence brain structure and cognitive function (Hashimoto et al., 2006; Liu et al., 2006; Porteous and Millar, 2006; Qu et al., 2007).

DISC1 encodes a full-length $\sim 100 \mathrm{kDa}$ protein (fl-DISC1) with various uncharacterized isoforms generated by alternative

\footnotetext{
Received April 3, 2007; revised July 18, 2007; accepted July 18, 2007.

This work was supported by Medical Research Council (United Kingdom) Grants G8604010 and G0400053 (M.D.H.) and G0600214 (D.J.P. and J.K.M.), Deutsche Forschungsgemeinschaft Grant KI1415/2 (E.K.), European Union Grant 037189 (E.K. and M.D.H.), and National Institutes of Health Grant R01-GM58553 (G.B.B.). J.K.M. is a UK Research Council Fellow.

*H.M. and S.M. are joint first authors.

Correspondence should be addressed to Dr. Miles D. Houslay, Professor, Molecular Pharmacology Group, Division of Biochemistry and Molecular Biology, Institute of Biomedical and Life Sciences, Wolfson Building, University of Glasgow, Glasgow G12 80Q, UK. E-mail: M.Houslay@bio.gla.ac.uk.

DOI:10.1523/JNEUROSCI.1493-07.2007

Copyright $\odot 2007$ Society for Neuroscience $\quad$ 0270-6474/07/279513-12\$15.00/0
}

splicing (Porteous and Millar, 2006). In brain, DISC1 is localized predominantly within regions implicated in the pathogenesis of psychiatric illness (Porteous and Millar, 2006). DISC1 acts as a scaffold protein, interacting with various proteins including those involved in neuronal migration (NUDEL and LIS1) and neurite extension (FEZ1) (Morris et al., 2003; Ozeki et al., 2003; Brandon et al., 2004; Miyoshi et al., 2004; Kamiya et al., 2005; Camargo et al., 2007; Shinoda et al., 2007; Taya et al., 2007), indicating a critical involvement in brain development and function.

cAMP plays a key role in many aspects of neuronal function and, recently, DISC1 has been shown to bind CAMP-specific phosphodiesterase-4B (PDE4B) (Millar et al., 2005). Four genes $(\mathrm{A} / \mathrm{B} / \mathrm{C} / \mathrm{D})$ encode the PDE4 family, with alternative mRNA splicing generating 20 isoforms (Conti et al., 2003; Houslay and Adams, 2003; Houslay et al., 2005). Long isoforms exhibit two conserved regions called UCR1 and UCR2, with UCR1 containing a site for stimulatory phosphorylation by protein kinase A (PKA) (Sette et al., 1994; MacKenzie et al., 2002).

The $P D E 4 B$ gene is an independent risk factor for psychiatric illness (Millar et al., 2005). Indeed, genetic variants within PDE4B (Pickard et al., 2007) and also within PDE4D (Tomppo et al., 
2006) associate with schizophrenia, suggesting a general involvement of PDE4 isoforms in conferring susceptibility to major mental illness. PDE4 genes are orthologous to Drosophila dunce, a gene involved in learning and memory (Davis et al., 1995). Moreover, PDE4-selective inhibitors exert potent antidepressant actions, whereas mice deficient in PDE4B and PDE4D behave as if on antidepressants (O'Donnell and Zhang, 2004; Zhang et al., 2005, 2006). Additionally, chronic nicotine treatment, which can exert antidepressant actions, selectively downregulates PDE4B in various brain regions (Polesskaya et al., 2007). Furthermore, PDE4-regulated cAMP signaling is of crucial importance for the protection, growth, and myelination of injured CNS axons (Gao et al., 2003; Nikulina et al., 2004; Pearse et al., 2004).

Here we analyze the $\sim 100 \mathrm{kDa}$ fl-DISC1 isoform to identify PDE4 subfamily-specific differences in interaction and cAMPmediated release from fl-DISC1. We show that increased cAMP levels cause release of PDE4D isoforms from fl-DISC1, whereas PDE4B isoforms remain stably associated. Insight into the basis of these selective actions comes from our use of a novel peptide array approach (Bolger et al., 2006; Baillie et al., 2007) to show that fl-DISC1 has two binding sites that PDE4 isoforms bind in common, together with additional N-terminal sites specific for PDE4B.

\section{Materials and Methods}

Materials. All materials for tissue culture were supplied by Sigma-Aldrich (Dorset, UK). L- $\left[{ }^{35} \mathrm{~S}\right]$-Methionine $(1000 \mathrm{Ci} / \mathrm{mmol}),\left[{ }^{32} \mathrm{P}\right]$ orthophosphate $(200 \mathrm{mCi} / \mathrm{mmol})$, and protein $\mathrm{G}-$ Sepharose beads were purchased from Amersham Biosciences (Buckinghamshire, UK). Anti-FLAG M2 monoclonal antibody, anti-maltose binding protein (MBP) antibody, anti-glutathione $S$-transferase (GST) antibody, forskolin, 3-isobutyl-1methylxanthine (IBMX), and epidermal growth factor (EGF) were from Sigma-Aldrich; anti-myc tag 9E10, anti- extracellular signal-regulated kinase 1/2 (Erk1/2), and anti-phospho-Erk1/2 antibodies were from Upstate (Paisley, UK); polyclonal anti-EPAC (exchange protein activated by cAMP) antibody was from Santa Cruz Biotechnology (Wiltshire, UK); EPAC agonist (8-pCPT-2'-O-Me-cAMP) was from Biolog (Bremen, Germany). Antisera specific for DISC1, PDE4A, PDE4B, PDE4C, and PDE4D have been described previously (Huston et al., 1996, 1997; Bolger et al., 1997; MacKenzie and Houslay, 2000; MacKenzie et al., 2002; James et al., 2004; Ogawa et al., 2005). Stearoyl DISC1 peptides were generated as described previously (Hundsrucker et al., 2006). These were stearoylSHSAFTSSFSFIRLSLGSAGERGE (CS1P) and stearoyl-IRSLTSEREGLEGLLSKLLVLSSRN (CS2P).

Mammalian expression constructs. Plasmids encoding FLAG-DISC1, mycHIS-fl-DISC1, mycHIS-( $\Delta 1-187)$-DISC1, PKA inhibitory peptide PKI, and PDE4 constructs PDE4A5, PDE4B1, PDEB2, PDEB3, PDE4C2, PDE4D3, PDE4D5, PDE4D3S54A, PDE4D3S54E, PDE4D3S13AS54A, and PDE4D-CAT have been described previously (Huston et al., 1996, 1997; Bolger et al., 1997; Owens et al., 1997; Hoffmann et al., 1998; Beard et al., 2000; Brandon et al., 2004; Fleming et al., 2004; Millar et al., 2005). DISC1 regions comprising residues $220-283$ and 359-854 were subcloned into $\mathrm{pEBG}-2 \mathrm{~T}$ vector to generate GST fusion proteins for expression in mammalian cells. A T744A site mutation in the FLAG-DISC1 ORF was introduced using the QuikChange II site-directed mutagenesis kit (Stratagene, Amsterdam, The Netherlands) according to the manufacturer's instructions with the following primers: T744A-sense $\left(5^{\prime}-\right.$ ACTCCGAGGATAAAAGGAAGGCCCCTTTGAAGGTATTGGAA-3' ) and T744A-antisense (5'-TTCCAATACCTTCAAAGGGGCCTTCCTTTTATCCTCGGAGT-3'). Mutagenesis was verified by sequencing before use.

Bacterial expression constructs. ORFs of PDE4B1 and PDE4D3 were subcloned into pMALC2 vector (New England Biolabs, Hertfordshire, UK) to generate N-terminal MBP fusion proteins. Similarly, the ORFs of PDE4B2 and individual PDE4D domains, UCR1, UCR2, and a catalytic (CAT) unit were subcloned into pGEX-5X-3 vector (Invitrogen, Paisley, UK) and expressed as N-terminal GST fusion proteins.
Cell culture and transient transfection. Cell lines HEK293, COS1, and SHSY5Y were maintained in DMEM supplemented with 10\% fetal calf serum (FCS), 10,000 U/ml penicillin/streptomycin, and $2 \mathrm{~mm}$ glutamine. Cell lines were transfected using Lipofectamine 2000 reagent (Invitrogen) according to recommended protocols. Forskolin, IBMX, EPAC agonist, and EGF were added to cultures at $100 \mu \mathrm{M}, 100 \mu \mathrm{M}, 10 \mu \mathrm{M}$, and 200 $\mathrm{ng} / \mathrm{ml}$, respectively, and incubated at $37^{\circ} \mathrm{C}$ for $15 \mathrm{~min}$ before harvesting of cells.

Immunoprecipitation and immunoblotting. Cells were washed with icecold PBS and lysed in PBS containing 1\% Triton X-100, 1 mm dithiothreitol (DTT), $10 \mathrm{~mm} \mathrm{NaF}$, and $5 \mathrm{~mm} \mathrm{NaPPi}$ with a protease inhibitor mixture added (Roche, West Sussex, UK). Lysates were solubilized by rotation on a rotary wheel for $30 \mathrm{~min}$ at $4^{\circ} \mathrm{C}$. Insoluble material was removed by a $15 \mathrm{~min}$ centrifugation at $14,000 \times g_{\text {av }}$ at $4^{\circ} \mathrm{C}$, followed by preclearing of lysed supernatants by incubation with protein $\mathrm{G}-\mathrm{Sepha}-$ rose beads for $30 \mathrm{~min}$ at $4^{\circ} \mathrm{C}$. Equalized amounts of precleared lysates were incubated with the indicated antibody for a minimum of $3 \mathrm{~h}$ at $4^{\circ} \mathrm{C}$, and immunocomplexes were captured after incubation with protein G-Sepharose beads for an additional 1-2 h. The immunoprecipitates were washed three times in lysis buffer and eluted from the beads by the addition of Laemmli buffer (LB) (Laemmli, 1970).

For immunoblotting, proteins were resolved by SDS-PAGE and transferred to nitrocellulose membranes. Membranes were blocked with 5\% low-fat milk powder in TBS (10 mm Tris- $\mathrm{HCl}, \mathrm{pH} 7.4$, and $150 \mathrm{~mm} \mathrm{NaCl}$ ) for $1 \mathrm{~h}$ at room temperature and incubated with the indicated primary antibody (in $1 \%$ low-fat milk powder, TBS, and $0.1 \%$ Tween 20 ) for $1 \mathrm{~h}$ at room temperature. Bound antibody was detected with the complementary horseradish peroxidase (HRP)-conjugated secondary antibody (Sigma-Aldrich), and proteins were visualized using enhanced chemiluminescence (Pierce, Northumberland, UK).

Confocal microscopy and immunofluorescence staining. Cells were transfected on coverslips in six-well plates and the following day were fixed in $4 \%$ paraformaldehyde in TBS containing 5\% sucrose for $10 \mathrm{~min}$ at room temperature. Cells were then permeabilized by performing three 5 min incubations with $0.2 \%$ Triton X-100/TBS, followed by three 5 min incubations with blocking buffer (10\% donkey serum and 2\% BSA). Coverslips were incubated for $2 \mathrm{~h}$ at room temperature with antisera specific for cAMP-activated EPAC at the required concentration in dilutant buffer (blocking buffer diluted 50\% with TBS). After three additional washes with blocking buffer, coverslips were incubated for $1 \mathrm{~h}$ with the complementary Alexa ${ }^{594}$-conjugated secondary antibody. After further washing with TBS, coverslips were mounted onto glass slides and observed using a Zeiss (Oberkochen, Germany) Pascal laser-scanning confocal microscope.

Treatment with DISC1 stearoyl peptides. Cells coexpressing fl-DISC1 with either PDE4D3 or PDE4B1 were pretreated with the peptides at a concentration of $50 \mu \mathrm{M}$ in $\mathrm{Me}_{2} \mathrm{SO}$ for 2-3 h before challenge with $100 \mu \mathrm{M}$ forskolin and $100 \mu \mathrm{M}$ IBMX for $15 \mathrm{~min}$. Control cells were pretreated with the equivalent concentration of $\mathrm{Me}_{2} \mathrm{SO}(0.2 \%)$.

Purification of MBP and GST fusion proteins. Bacterial expression constructs were transformed into Escherichia coli BL21 cells. A $10 \mathrm{ml}$ starter culture was used to inoculate $400 \mathrm{ml}$ of LB medium containing 100 $\mu \mathrm{g} / \mathrm{ml}$ ampicillin and was grown at $37^{\circ} \mathrm{C}$ until the culture reached an $A_{600}$ of 0.5 . Expression of the fusion proteins was induced by the addition of 1 $\mathrm{mm}$ isopropyl- $\beta$,D-thiogalactopyranoside for $4 \mathrm{~h}$ before cells were harvested by centrifugation at $10,000 \times g_{\text {av }}$ for $15 \mathrm{~min}$ at $4^{\circ} \mathrm{C}$. Pellets were then lysed in $20 \mathrm{ml}$ of STE buffer ( $10 \mathrm{~mm}$ Tris- $\mathrm{HCl}$, pH 8.0, $150 \mathrm{~mm} \mathrm{NaCl}$, and $1 \mathrm{~mm}$ EDTA) containing $1 \mathrm{mg} / \mathrm{ml}$ lysozyme and incubated with rotation at $4^{\circ} \mathrm{C}$ for $1 \mathrm{~h}$. DTT to a final concentration of $5 \mathrm{~mm}$ and $10 \%$ Sarkosyl in STE buffer to a final concentration of $1.5 \%$ were added to lysates before sonication for $1 \mathrm{~min}$. The removal of insoluble material was performed by a $15 \mathrm{~min}$ centrifugation at $10,000 \times g_{\text {av }}$ at $4^{\circ} \mathrm{C}$. Ten percent Triton X-100 in STE buffer to a final concentration of $2 \%$ and a mixture of protease inhibitors were added to the cleared supernatants and stored at $-80^{\circ} \mathrm{C}$ until used.

MBP and GST fusion protein-cleared lysates were added to $500 \mu \mathrm{l}$ of amylose resin (New England Biolabs) and $500 \mu$ of glutathione-Sepharose beads (Amersham Biosciences), respectively, and incubated for $2 \mathrm{~h}$ at $4^{\circ} \mathrm{C}$. Beads were pelleted by centrifugation $\left(10,000 \times g_{\text {av }}\right.$ for $\left.1 \mathrm{~min}\right)$ and 
A

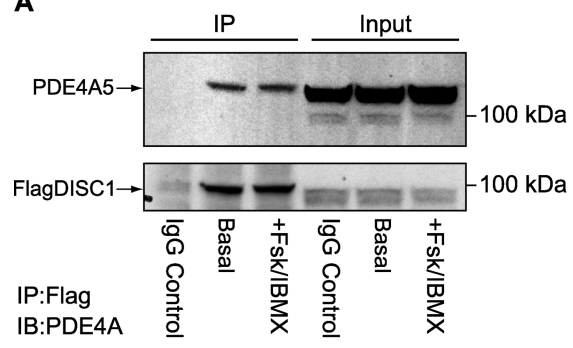

C

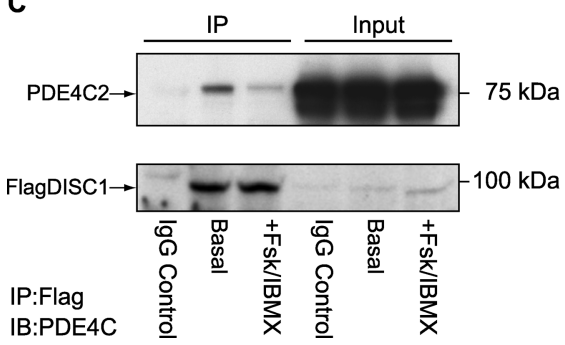

B

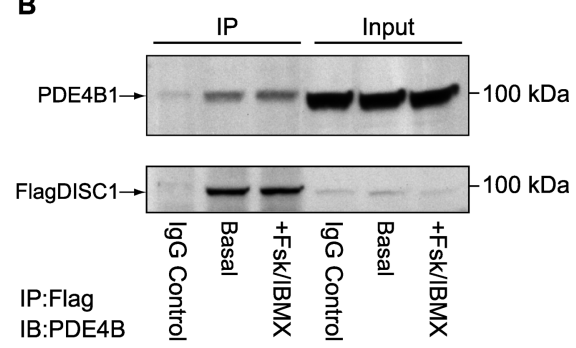

D

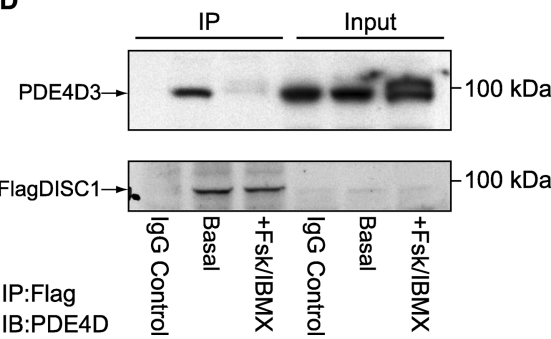

Figure 1. Interaction of the fl-DISC1 isoform with PDE4 isoforms from all four PDE4 gene families. An N-terminally FLAGtagged form of the fl-DISC1 isoform (FlagDISC1) was coexpressed in HEK293 cells with PDE4 isoform PDE4A5 (A), PDE4B1 (B), PDE4C2 (C), or PDE4D3 (D). Cells were challenged with vehicle or $100 \mu \mathrm{m}$ forskolin (Fsk)/100 $\mu \mathrm{m} \mathrm{IBMX} \mathrm{for} 15$ min before harvesting. Equalized amounts of cell lysate were immunoprecipitated (IP) with either control IgG or M2 anti-FLAG antibody and immunoblotted (IB) with PDE4A (A), PDE4B (B), PDE4C (C), or PDE4D (D) antisera (top). Equal immunocapture of FlagDISC1 was determined by immunoblotting with the anti-FLAG M2 antibody (bottom). The relative expression levels of FlagDISC 1 and PDE4 isoforms in $\sim 5 \%$ of total cell lysate input used for coimmunoprecipitation assays were determined by direct immunoblotting with the PDE4 antisera (top) and the anti-FLAG M2 antibody (bottom).

washed three times in $\mathrm{PBS} / 1 \%$ Triton $\mathrm{X}-100$ with protease inhibitors added. The MBP fusion proteins were eluted from the beads by three incubations of $30 \mathrm{~min}$ at $4^{\circ} \mathrm{C}$ with $600 \mu \mathrm{l}$ of STE buffer plus $10 \mathrm{~mm}$ maltose. Using a similar protocol, GST fusion proteins were eluted with $30 \mathrm{~mm}$ glutathione in STE buffer. The eluted fractions were pooled and dialyzed for at least $18 \mathrm{~h}$ at $4^{\circ} \mathrm{C}$ against three changes of dialysis buffer $(20$ $\mathrm{mm}$ Tris- $\mathrm{HCl}, \mathrm{pH} 7.6$, and $50 \mathrm{~mm} \mathrm{NaCl} ; 2 \mathrm{~L}$ total volume) before storage at $-80^{\circ} \mathrm{C}$.

In vitro protein synthesis. $\left[{ }^{35} \mathrm{~S}\right]$-labeled FLAG-DISC1 protein was synthesized using the T7 transcription/translation-coupled reticulocyte lysate system (Promega, Hampshire, UK). The translation product was partially purified by ammonium sulfate precipitation as described previously (Bardwell et al., 1992) before use in peptide array analyses.

Spot synthesis of peptides and overlay experiments. Peptide libraries were produced by automatic Spot synthesis as described by us previously (Bolger et al., 2006; Baillie et al., 2007). Interaction of peptide spots with MBP- or GST-fused purified proteins was determined by overlaying the cellulose membranes with $10 \mu \mathrm{g} / \mathrm{ml}$ recombinant protein. Bound recombinant proteins were detected with specific primary antisera and a complementary HRP-coupled secondary antibody as for immunoblotting. For PDE4 arrays, membranes were incubated with translated ${ }^{35} \mathrm{~S}$-labeled FLAG-tagged fl-DISC1 protein that had been partially purified from 500 $\mu \mathrm{l}$ of reticulocyte lysate. Binding of $\left[{ }^{35} \mathrm{~S}\right]$-FLAG-fl-DISC1 was detected by phosphorimager analysis (Bio-Rad, Hertfordshire, UK).

Metabolic labeling and in vivo phosphorylation of FLAG-DISC1. Cells transfected with the FLAG-tagged fl-DISC1 cDNA were subdivided into six-well culture dishes $24 \mathrm{~h}$ after transfection and cultured for an additional day. Cell medium was aspirated, followed by washing in phosphate-free DMEM (PFDMEM), and incubated overnight with 350 $\mu \mathrm{l} /$ well of labeling medium containing PFDMEM, 2\% FCS, $20 \mathrm{~mm}$ HEPES, pH 7.4, and $100 \mu \mathrm{Ci}$ of $\left[{ }^{32} \mathrm{P}\right]$ orthophosphate. Cells were then treated for $15 \mathrm{~min}$ with vehicle or $100 \mu \mathrm{M}$ forskolin/100 $\mu \mathrm{M}$ IBMX before harvesting on ice. The labeling medium was discarded, and cells were washed twice with ice-cold PBS and lysed in immunoprecipitation lysis buffer supplemented with $10 \mu \mathrm{M}$ okadaic acid. Immunoprecipitated radiolabeled FLAG-tagged fl-DISC1 was visualized by phosphorimager analysis.

\section{Results}

DISC1 has previously been shown to interact with members of the PDE4 family through their conserved UCR2 domain (Millar et al., 2005). In SHSY5Y cells, the endogenous $71 \mathrm{kDa}$ DISC1 isoform binds PDE4B, which is subsequently released in a PKA-phosphorylated, activated state in response to increased cellular cAMP levels (Millar et al., 2005). Investigation of DISC1-PDE4 interactions is extended in the present study by examining interaction of the fl-DISC1 isoform in immunopurified complexes from both HEK293 and SHSY5Y cells.

\section{Coimmunoprecipitation of PDE4 with fl-DISC1}

Exogenous fl-DISC1 coimmunoprecipitates with PDE4 isoforms from each of the four PDE4 gene families (namely PDE4A5, PDE4B1, PDE4C2, and PDE4D3) when these species are cotransfected in HEK293 cells (Fig. 1). This is consistent with our previous work and the notion that PDE4 isoforms share a common binding site within DISC1 (Millar et al., 2005).

To evaluate whether these different PDE4 isoforms could be released from flDISC1 in response to elevated CAMP levels, HEK293 cells were treated with a combination of forskolin plus the nonspecific phosphodiesterase inhibitor IBMX, to maximize cAMP levels within the cells. Intriguingly, although such treatment had no effect on fl-DISC1 coimmunoprecipitation with either PDE4A5 or PDE4B1 (Fig. $1 A, B$ ), the level of either PDE4D3 or PDE4C2 associated with fl-DISC1 was markedly reduced (Fig. 1C,D). Such results contrast with previous observations with the $71 \mathrm{kDa}$ DISC1 isoform, where PDE4B was released in response to increased intracellular cAMP concentrations in SHSY5Y cells (Millar et al., 2005). Our investigations here, in which we evaluate fl-DISC1, suggest that PDE4-DISC1 interactions may be more subtle than originally anticipated and seemingly determined by isoform differences in both DISC1 and PDE4.

We thus set out to examine in more detail the interaction of both PDE4D3 and PDE4B1 with fl-DISC1 as examples of isoforms that respond differentially to elevation of intracellular cAMP levels. With this in mind, we wanted to determine whether such differences were merely specific to these PDE4 isoforms or a more general feature attributable to these two PDE4 subfamilies. As with the PDE4B1 long isoform, the PDE4B2 short form and the PDE4B3 long form both coimmunoprecipitate with fl-DISC1 and are not released after treating cells with forskolin/IBMX to increase cAMP levels in HEK293 cells (Fig. 2A). In contrast to this, although the long PDE4D5 isoform also coimmunoprecipitated with fl-DISC1, it, like PDE4D3, was released after treating cells with forskolin/IBMX to increase cAMP levels (Fig. $2 B$ ).

We also investigated these interactions in transfected SHSY5Y cells as a model for a neuronal cell environment. In such a system, we observed that in response to forskolin/IBMX challenge, as in HEK293 cells, PDE4B1 and fl-DISC1 did not dissociate, whereas PDE4D3 and fl-DISC1 did dissociate (Fig. 2C). Consistent with 
these data, generated using exogenously overexpressed proteins, we also demonstrated that endogenous $100 \mathrm{kDa}$ PDE4B complexes isolated from SHSY5Y cells remained stably associated after treatment with forskolin/IBMX (Fig. 2D).

\section{Probing fl-DISC1 peptide arrays with PDE4D3 and PDE4B1 fusion proteins} These data suggest that attenuation of flDISC1 interaction with PDE4 isoforms by increased cAMP levels is PDE4 subfamily dependent and therefore likely involves distinct interaction sites. Spotimmobilized peptide array analysis has proved to be a powerful novel technology to gain insight into putative interaction sites underpinning protein-protein interaction. Indeed, we have recently exploited this technique to identify interaction sites that define the interaction of the PDE4D5 isoform with the signaling scaffold proteins $\beta$ arrestin and RACK1 (Bolger et al., 2006; Baillie et al., 2007) and between PDE4D3 and the PKA scaffold AKAP18 $\delta$ (Stefan et al., 2007). Taking PDE4D3 and PDE4B1, which are both expressed in brain (Engels et al., 1995; Bolger et al., 1997; Thompson et al., 2000; Miro et al., 2002; Richter et al., 2005), as paradigms for classes of PDE4 isoforms that show profound differences in susceptibility to release from fl-DISC1 after elevation of intracellular cAMP, we set out to see whether they showed differences in interaction with a scanning peptide array of fl-DISC1. In this strategy, a library of overlapping peptides (25-mers), sequentially shifted by 5 aa across the entire sequence of fl-DISC1, was immobilized on cellulose membranes. This was then probed with either MBPPDE4B1 or MBP-PDE4D3, which are recombinant fusion proteins of PDE4B1 and PDE4D3, respectively, made with $\mathrm{MBP}$ and expressed in E. coli before purification for use as probes. Here we show portions of typical arrays in which positive interactions are evident as dark spots on the array (Fig. $3 A, B$ ). Both of the probes identified two interaction sites on the fl-DISC1 arrays, corresponding to amino acids 190-230 [PDE4 common site 1 (cs1)] and 611-650 (PDE4 cs2) in fl-DISC1 (Fig. 3A, C), indicating that they are likely to provide common sites of interaction for these PDE4 isoforms. In this regard, previous studies (Millar et al., 2005), using truncated DISC1 constructs, had identified a region overlapping with $\mathrm{PDE} 4 \mathrm{cs} 1$ (Fig. $3 C$ ) as of importance in PDE4B binding. Additionally, however, we show here that the PDE4B1 probe, but not the PDE4D3 probe, interacted with three additional regions on the fl-DISC1 peptide array (Fig. $3 B, C$ ) corresponding to residues $31-65$ [PDE4B specific site 1 (4bss1)], 101-135 (4bss2), and 266-290 (4bss3). These additional interaction sites on the fl-DISC1 array were also detected using a PDE4B2 probe (GST-PDE4B2), indicating that interaction was PDE4B subfamily specific (Fig. 3D).

We set out to confirm independently that PDE4B isoforms
B
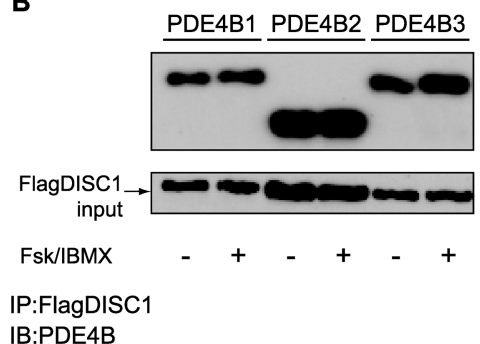

IB:PDE4B

D

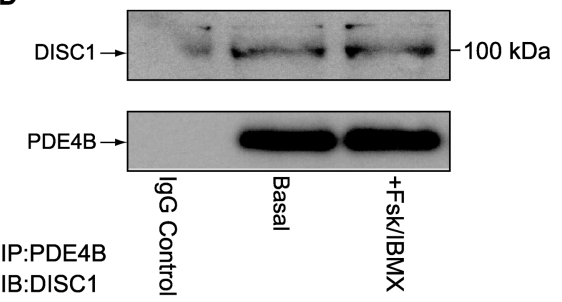

Figure 2. PDE4B isoforms remain stably associated with the fl-DISC1 isoform; DISC1 interaction with members of the PDE4D subfamily are disrupted by treatment with forskolin/IBMX. $A$, HEK293 cells were cotransfected to express Flag-tagged fl-DISC1 d PDE4B isoforms PDE4B1, PDE4B2, and PDE4B3. Cells were challenged by treatment with vehicle or $100 \mu \mathrm{m}$ forskolin/100 $\mu \mathrm{m}$

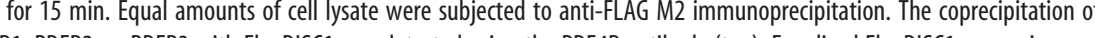
.

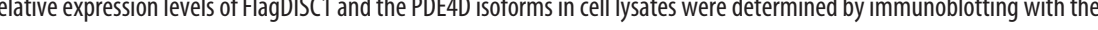
DISC1 and either PDE4B1 or PDE4D3. Cells were treated with or without $100 \mu \mathrm{m}$ forskolin/100 $\mu \mathrm{m}$ IBMX for 15 min before forskolin/100 $\mu \mathrm{M}$ IBMX for 15 min before preparation of cell lysates. Anti-PDE4B immunoprecipitation was performed to isolate endogenous PDE4B-DISC1 complexes. The amount of PDE4B (bottom) and DISC1 (top) present in PDE4B immunoprecipitates was determined by immunoblotting. An antibody that specifically recognizes the $100 \mathrm{kDa}$ fl-DISC1 isoform was used ( $0 \mathrm{gawa}$ et al., 2005). Fsk, Forskolin; IP, immunoprecipitated; IB, immunoblotted.

interact with both the $\mathrm{N}$ - and C-terminal regions of DISC1 using truncated forms of fl-DISC1. Thus, both a GST-DISC1 (amino acids 220-283) fusion protein that encompasses the PDE4 cs1 and $4 \mathrm{bss} 3$ binding sites as well as a GST-DISC1 (amino acids 359-854) fusion protein encompassing the PDE4 cs2 (Fig. 3E) were able to bind to PDE4B when coexpressed in transfected HEK293 cells (Fig. 3E).

Very recently, novel point mutations in the murine Discl gene have been identified that confer behavioral, brain structural, and pharmacological phenotypes related to schizophrenia and depression (Clapcote et al., 2007). These are predicted to cause, individually, Gln31Leu and Leu100Pro mutations in murine Disc1. Intriguingly, such mutations are located within regions that we have implicated here as being involved specifically in PDE4B association with DISC1, namely $4 \mathrm{bss} 1$ and $4 \mathrm{bss} 2$, respectively (Fig. 3C). Because sequences surrounding Gln31 and Leu100 in murine Disc1 are not highly conserved in human DISC1 (Clapcote et al., 2007), we used peptide Spot arrays to determine whether PDE4B would interact with peptides that in- 


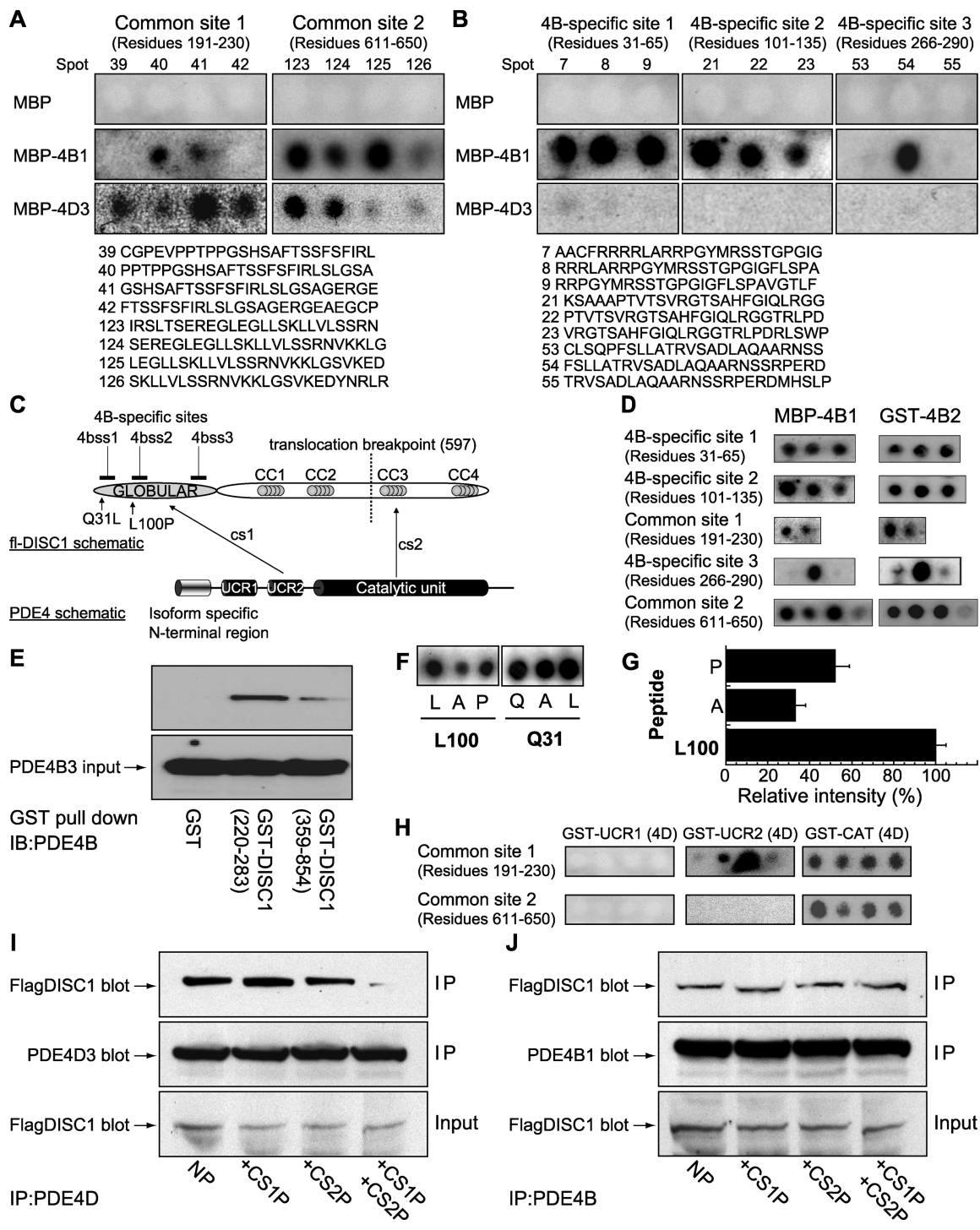

Figure 3. Probing DISC1 peptide arrays for PDE4B and PDE4D interaction sites. $A, B$, An array of immobilized peptide spots of overlapping 25 -mer peptides, each shifted along by 5 a a in the entire sequence of the fl-DISC 1 is oform, was probed for interaction with MBP fusion proteins of PDE4B1 (MBP-4B1) or PDE4D3 (MBP-4D3) or MBP alone and detected by immunoblotting. Dark spots were evidence of positive interaction, whereas noninteracting peptides left white (blank) spots. In all other sections of the array, spots were blank with the probes. Spot numbers relate to the 25 -mer peptides in the array with the amino acid sequence of the peptide spots given below the array data. C, Schematic of DISC1 and PDE4 protein structure. DISC1 comprises an N-terminal globular domain with predicted coiled-coil (CC) regions within its C-terminal domain. Long PDE4 isoforms consist of two upstream conserved regions (UCR1 and UCR2) linked to the CAT subunit by linker regions. Distinct PDE4 isoforms are characterized by a unique N-terminal region. The sites of DISC1-PDE4 interaction established from peptide array analyses are highlighted (CS, common PDE4 binding site; 4bss, PDE4B-specific binding site). The relative position of mutations in mouse Disc 1 that confer phenotypes related to psychiatric illness are indicated, namely Q31L and L100P D, DISC1 peptide arrays were probed with either MBP-4B1 or a GST fusion protein of PDE4B2 (GST-4B2), and interaction was detected by immunoblotting. Positively interacting peptide spots are shown with the related DISC1 regions given. $\boldsymbol{E}$, HEK293 cells were transfected with the indicated pEBG-2T DISC1 plasmids and FLAG-tagged PDE4B3. Lysates were incubated with glutathione-Sepharose beads to capture GST or GST-fl-DISC1 complexes and immunoblotted with the anti-GST antibody to detect fl-DISC1 fusion proteins (data not shown) or anti-FLAG M2 antibody to detect the presence of coprecipitated PDE4B3. $\boldsymbol{F}$, An MBP fusion protein of PDE4B1 was used to probe peptides reflecting the murine Disc1 sequence, namely one containing GIn31 (Q31), GHGLPPAVAPQ(31)RRRLTRRPGYMRST (left) and one containing Leu100 (L100), SHCQASLVGKPFL(100)KSSLVPAVASEG (right). In addition the indicated mutant peptides were probed to assess PDE4B1 binding to Leu100Ala, Leu100Pro, Gln31Ala, and Gln31Leu. G, Densitometric analysis of MBP-PDE4B1 interaction with the wild-type and indicated mutant forms of the Leu100 SHCOASLVGKPFL(100)KSSLVPAVASEG peptide. $\boldsymbol{H}$, GST fusion proteins of individual PDE4D domains, UCR1, UCR2, and CAT were used to probe fl-DISC1 peptide arrays and binding of probes detected by immunoblotting with the anti-GST antibody. The interactions of the PDE4D probes with fl-DISC1 peptide spots of the common PDE4 binding sites (as determined in Fig. 4A) are shown. I, HEK293 cells coexpressing Flag-tagged fl-DISC1 and PDE4D3 were incubated for $2-3 \mathrm{~h}$ with $50 \mu \mathrm{m}$ stearoylated DISC 1 peptides of DISC 1 regions encompassing cs 1 (CS1P, SHSAFTSSFSFIRLSLGSAGERGE) and cS2 (CS2P, IRSLTSEREGLEGLLSKLLVLSSRN). Cells were then harvested, and lysates were immunoprecipitated with PDE4D antisera. Coprecipitation of FlagDISC1 was assessed by immunoblotting with the anti-FLAG M2 antibody (top). Equal immunocapture of PDE4D3 was determined by immunoblotting immunoprecipitates with the PDE4D antibody (middle). The cluded these sites of mutation in murine Disc1. Indeed, we show here that the MBP fusion protein of PDE4B1 interacted with each of the murine Discl peptides that, respectively, both encompass these mutations and the cognate human sequence of which is located in either $4 \mathrm{bss} 1$ or $4 \mathrm{bss} 2$ sites (Fig. 3F). Furthermore, we clearly observed a diminished interaction with the Leu100-containing peptide when this single residue was substituted with either Leu100Ala or Leu100P (Fig. $3 F, G$ ), the latter mutation leading to reduced PDE4B1/Disc1 association in mutant mice (Clapcote et al., 2007). In contrast, Gln31Ala and Gln31Leu substitutions, found in mutant mice (Clapcote et al., 2007), did not reduce the interaction with the murine peptide that reflected sequence at the $4 \mathrm{bss} 1$ site (Fig. $3 F$ ). This suggests that mutation at such a site likely exerts its effect by altering the conformation of Disc1, thereby indirectly affecting interaction with PDE4B.

\section{Probing fl-DISC1 peptide arrays with} UCR1, UCR2, and the PDE4D CAT unit Individual PDE4 isoforms differ by virtue of their extreme $\mathrm{N}$-terminal regions, with the common regions being provided by UCR1, UCR2, and the CAT unit. $\mathrm{N}$-terminal truncation studies have previously implicated UCR2 as of importance in the interaction of PDE4B with DISC1 (Millar et al., 2005). Here we have suggested that PDE4B and PDE4D likely contain similar binding sites able to bind to common sites on fl-DISC1, which may be represented by PDE4 cs1 and PDE4 cs2 (Fig. 3C), whereas PDE4B likely contains additional binding sites able to interact with the regions encompassed by $4 \mathrm{bss} 1-3$ (Fig. 3C) on fl-DISC1. To gain insight into which regions of PDE4D were involved in interacting with fl-DISC1, we probed the fl-DISC1 array with recombinant GST fu-

\section{$\leftarrow$}

relative expression level of FlagDISC1 in $\sim 5 \%$ of total cell lysate input used for coimmunoprecipitation assays was determined by direct immunoblotting with the anti-FLAG M2 antibody (bottom). $J$, As in $I$, cells coexpressing Flag-tagged fl-DISC1 and PDE4B1 were incubated with stearoylated DISC1 peptides CS1P and CS2P. Cell lysates were then incubated with PDE4B antisera, and PDE4B immunoprecipitates were immunoblotted with anti-FLAG M2 antibody to detect coprecipitated FlagDISC1 (top). Immunocapture of PDE4B1 was confirmed by immunoblotting immunoprecipitates with PDE4B antisera (middle). Equivalent input expression of FlagDISC 1 was determined by immunoblotting $\sim 5 \%$ of total cell lysate used for the coimmunoprecipitation assays with the anti-FLAG M2 antibody (bottom). IP, Immunoprecipitated; IB, immunoblotted. 
sion proteins of either UCR1 or UCR2 or the full-length CAT unit of PDE4D. No positive binding spots were detected with GST-UCR1 overlay, whereas with GSTUCR2, positive (dark) spots were evident in peptides of the first common binding region, cs1 (Fig. 3H). These data are consistent with previous $\mathrm{N}$-terminal truncation studies implying that UCR2, and not UCR1, played a role in the binding of PDE4B1 to DISC1 (Millar et al., 2005). This is also consistent with studies using full-length MBP-PDE4D3 and MBPPDE4B1 as probes (Fig. $3 A, B$ ), which each identified PDE4 cs1 (Fig. $3 C$ ) as a possible (common) interaction region in fl-DISC1. This is, presumably, attributable to an interaction involving the common 80 aa UCR2 that is highly conserved (93\%) between PDE4D and PDE4B (Conti et al., 2003; Houslay and Adams, 2003; Houslay et al., 2005).

The PDE4D CAT unit fusion protein identified the second common interaction site, PDE4 cs2 (Fig. 3C,H), suggesting that UCR2 and the CAT unit provide the sources of interaction with two PDE4 subfamily common interaction sites in flDISC1. Notwithstanding this, the GSTCAT fusion protein also appeared to interact with peptide spots representing the PDE4 cs1 region (Fig. 3C,H). A possible reason for this is an intriguing shared motif of the form $\phi$-Ser- $\mathrm{X}_{2}-\phi$ - $\mathrm{X}_{2}$-Ser-Ser$\mathrm{X}_{2}-\phi$-X-(Lys/Arg)-Leu- $\mathrm{X}_{0 / 1}-$ Ser- $\phi$ - $\mathrm{X}_{5}$ -

Arg, observable within both $\operatorname{cs} 1$ and $\operatorname{cs} 2(\phi$ is a hydrophobic amino acid).

These data imply that PDE4D3 interacts with fl-DISC1 only through cs1 and cs2, whereas PDE4B1 interaction with fl-DISC1 involves additional binding sites. We thus set out to determine whether the binding of PDE4D3 could be disrupted in cells using peptides that represent the common core cs1 and cs2 regions and thus would be expected to compete with fl-DISC1 for sequestering PDE4D3. Stearoylated peptides have been shown to enter cells, and specific species have been used to disrupt protein-protein complexes by competing for binding partners (Hundsrucker et al., 2006). Thus, we treated cells transfected to express both flDISC1 and PDE4D3 with either or both peptide CS1P (DISC1 residues 201-SHSAFTSSFSFIRLSLGSAGERGE-224), which reflects the cs1 region, and peptide CS2P (DISC1 residues 611IRSLTSEREGLEGLLSKLLVLSSRN-635), which reflects the cs2 region to compete for PDE4 interaction at these common core sites in fl-DISC1. Here we see that treatment with either CS1P or CS2P peptide alone had no effect on PDE4D3-fl-DISC1 interaction, as evidenced from coimmunoprecipitation studies, when cells were treated with both peptides together and then PDE4D3 interaction with fl-DISC1 was abolished (Fig. 3I). In marked contrast to this, exposure to CS1P or CS2P peptides either alone or together failed to disrupt PDE4B1-fl-DISC1 interaction (Fig. $3 J)$, consistent with additional sites being involved in PDE4B1fl-DISC1 interaction. regions highlighted.
B

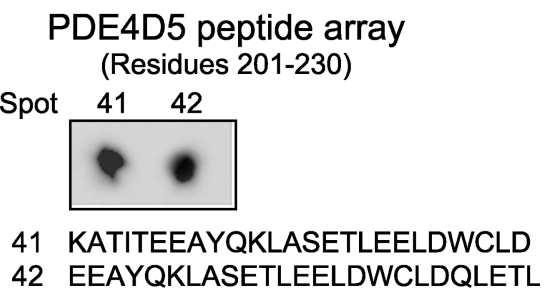

42 EEAYQKLASETLEELDWCLDQLETL

PDE4B sequence

PDE4D sequence

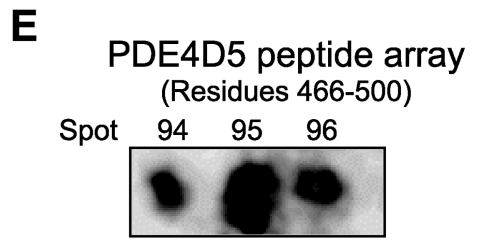

94 VLENHHLAVGFKLLQEENCDIFQNL 95 HLAVGFKLLQEENCDIFQNLTKKQR 96 FKLLQEENCDIFQNLTKKQRQSLRK

Figure 4. Probing PDE4B and PDE4D peptide arrays for fl-DISC1 interaction sites: PDE4 subfamilies exhibit common regions of DISC1 binding. $\boldsymbol{A}, \boldsymbol{B}, \boldsymbol{D}, \boldsymbol{E}$, Peptide arrays of the $\operatorname{PDE} 4 \mathrm{~B}(\boldsymbol{A}, \boldsymbol{D})$ and $\operatorname{PDE} 4 \mathrm{D}(\boldsymbol{B}, \boldsymbol{E})$ sequences were probed with partially purified ${ }^{35}$ S]-labeled fl-DISC1 protein, and binding to peptide spots was detected by phosphorimager analysis. $C$, $\boldsymbol{F}$, Sites common to both PDE4B and PDE4D for fl-DISC1 interaction are shown with the sequence homology between PDE4B and PDE4D at these binding

Probing PDE4D and PDE4B peptide arrays with fl-DISC1 We set out now to perform the converse experiments probing PDE4D and PDE4B peptide arrays with fl-DISC1. Unfortunately, as with others previously, despite much effort with different growth conditions and constructs, we were singularly unsuccessful in generating fl-DISC1 as a soluble protein able to be purified from $E$. coli when expressed as a GST fusion protein. To generate a useful probe, we therefore expressed fl-DISC1 as a radiolabeled species using an in vitro transcription-translation system in which $\left[{ }^{35} \mathrm{~S}\right]$ methionine was incorporated into the protein. We have used a similar strategy previously to generate $\left[{ }^{35} \mathrm{~S}\right]$-labeled PDE4A1 for various binding studies (Smith et al., 1996; Baillie et al., 2002). Using a cDNA encoding fl-DISC1 as a template, we identified a single radiolabeled protein product of the expected size $(100 \mathrm{kDa})$ in extracts analyzed on SDS-PAGE (data not shown). This product was used for overlays of PDE4 peptide arrays, with detection by phosphorimager analysis.

Analysis of PDE4B and PDE4D arrays revealed DISC1 interacted with two common regions that corresponded, respectively, to residues within the UCR2 domain (Fig. $4 A, B$ ) and CAT unit (Fig. $4 D, E$ ). Alignment between the UCR2 domains of PDE4D and PDE4B show an overall 90\% sequence identity (supplemental Fig. 1, available at www.jneurosci.org as supplemental material), whereas the CAT unit sequences of PDE4D and PDE4B 
A

PDE4B1 peptide array (Residues 352-380)

Spot

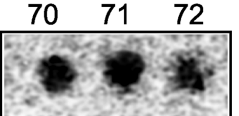

70 IFNVAGYSHNRPLTCIMYA

71 GYSHNRPLTCIMYAIFQER

72 RPLTCIMYAIFQERDLLKT

B

PDE4D5 peptide array

(Residues 341-375)

Spot $\quad 69 \quad 70 \quad 71$

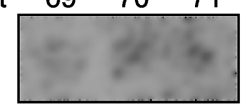

69 GLHVFRIAELSGNRPLTVIMHTIFQ

70 RIAELSGNRPLTVIMHTIFQERDLL

71 SGNRPLTVIMHTIFQERDLLKTFKI
C

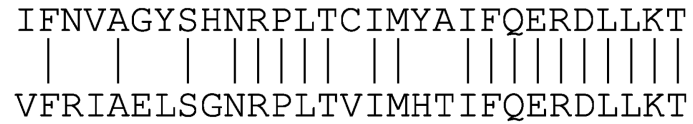

D

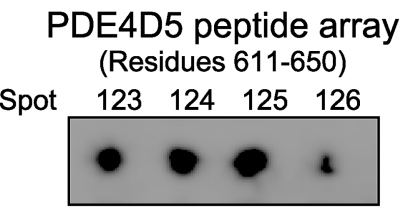

123 IDYIVHPLWETWADLVHPDAQDILD

124 HPLWETWADLVHPDAQDILDTLEDN

125 TWADLVHPDAQDILDTLEDNREWYQ

126 VHPDAQDILDTLEDNREWYQSTIPQ

$\mathbf{F}$

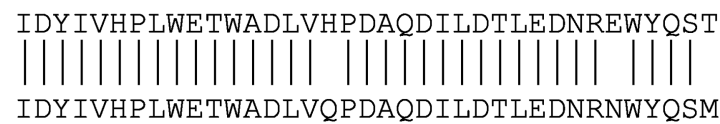

PDE4D sequence

PDE4B sequence

Figure 5. Probing PDE4B and PDE4D peptide arrays for DISC1 interaction sites: PDE4B- and PDE4D-specific sites of DISC1 binding. $\boldsymbol{A}, \boldsymbol{B}, \boldsymbol{D}, \boldsymbol{E}$, As in Figure 4, PDE4B $(\boldsymbol{A}, \boldsymbol{E})$ and PDE4D $(\boldsymbol{B}, \boldsymbol{D})$ peptide arrays were probed with [ $\left.{ }^{35} \mathrm{~S}\right]-\mathrm{fl}-\mathrm{DISC}$. $\boldsymbol{C}, \boldsymbol{F}$, Sites of $\left[{ }^{35} \mathrm{~S}\right]-$ DISC 1 binding unique to PDE4B or PDE4D are shown with comparative PDE4B and PDE4D sequence alignment at these sites highlighted.

show an overall 85\% identity (supplemental Fig. 1, available at www.jneurosci.org as supplemental material). Comparative sequence analysis shows that each of the fl-DISC1 binding regions is highly conserved between PDE4B and PDE4D (Fig. 4C,F).

In addition to these two common regions, radiolabeled $\mathrm{fl}-$ DISC1 bound to a region specific to the PDE4B CAT unit (Fig. $5 A, B)$ and, intriguingly, one specific for the PDE4D CAT unit (Fig. 5D,E). Alignment of PDE4B and PDE4D sequences within these regions highlight significant disparities in the primary structure (Fig. 5C,F).

\section{N-terminal truncation of DISC1 allows cAMP-mediated release of PDE4B1}

Increased cAMP levels cause the release of PDE4D3, but not PDE4B1, from fl-DISC1. Our peptide array analyses indicate that the N-terminal portion of fl-DISC1 contains three potential sites involved in facilitating PDE4B, but not PDE4D, interaction. We hypothesize that elevated cAMP levels disrupt PDE4 interaction at the common binding sites on DISC1 and that PDE4B is protected from release as it also interacts with additional binding sites on fl-DISC1 that are contained within its $\mathrm{N}$-terminal region. To explore this, we generated a truncate of fl-DISC1 that lacks the first $187 \mathrm{~N}$-terminal amino acids $(\Delta 1-187-f l-D I S C 1)$ and that removes two of the three putative PDE4B-unique binding regions. We show here that this construct still binds PDE4B1, but now, as with
PDE4B sequence

PDE4D sequence
PDE4D3, it is released during elevation of intracellular cAMP levels (Fig. 6).

PKA action is required for the release of PDE4D3 from fl-DISC1

The cellular phenotypes consequent after cAMP elevation are transduced through the activation of either PKA (Tasken and Aandahl, 2004) or EPAC (Bos, 2006), a guanine-nucleotide exchange protein that is directly activated by cAMP. Here we show that coimmunoprecipitation of PDE4D3 with fl-DISC1 was unaffected when either untransfected cells or those transfected to allow for exogenous EPAC1 expression were treated with EPAC agonist (Fig. 7A). Activation of EPAC1 was confirmed by confocal imaging of cells immunostained with antisera raised specifically against the cAMP-activated form of EPAC1 (data not shown). In contrast, coimmunoprecipitation of PDE4D3 with flDISC1 was markedly attenuated after forskolin/IBMX treatment (Figs. 1D, 7A). These data indicate a role for PKA rather than EPAC in underpinning PDE4D3 release from fl-DISC1. Consistent with such an involvement of PKA, the action of forskolin/ IBMX was attenuated after expression of the PKA inhibitory peptide PKI (Fig. 7B).

Because learning and memory pathways and defects in schizophrenia can involve the ERK signaling pathway (Kyosseva, 2004; Sweatt, 2004) as well as that directed by cAMP, we set out to determine whether ERK activation, which occurs consequent after EGF challenge of these cells, might alter the association of PDE4D3 with fl-DISC1. However, activation of ERK during stimulation with EGF failed to dissociate DISC1-PDE4D3 complexes (Fig. 7C). Immunoblotting of cell lysates with phosphoErk1/2 antisera confirmed the activation of the Erk signaling pathway in these cells after EGF challenge (Fig. 7C).

PKA phosphorylation of PDE4D3 is not required for attenuation of PDE4D3 interaction with fl-DISC1

Activation of PKA by increased cAMP levels causes the phosphorylation of long isoforms from all four PDE4 subfamilies at a conserved site within UCR1, leading to their activation (Sette et al., 1994; Sette and Conti, 1996; Hoffmann et al., 1998; MacKenzie et al., 2002). In the case of PDE4D3, such stimulatory phosphorylation occurs at Ser54 within the PKA consensus motif RRES. We thus used mutants at Ser54 to evaluate whether phosphorylation at this site was involved in the cAMP-dependant attenuation of fl-DISC1-PDE4D3 interaction. Here we see that the Ser54Ala mutant of PDE4D3, which can no longer be phosphorylated at this site as shown previously (Sette et al., 1994; Sette and Conti, 1996; Hoffmann et al., 1998; MacKenzie et al., 2002), is still released after elevating cAMP levels by forskolin/IBMX challenge (Fig. 8A). The Ser54Asp mutant has previously been shown to mimic the stimulatory effect of PKA phosphorylation at this site in PDE4D3. However, the Ser54Asp-PDE4D3 mutant 


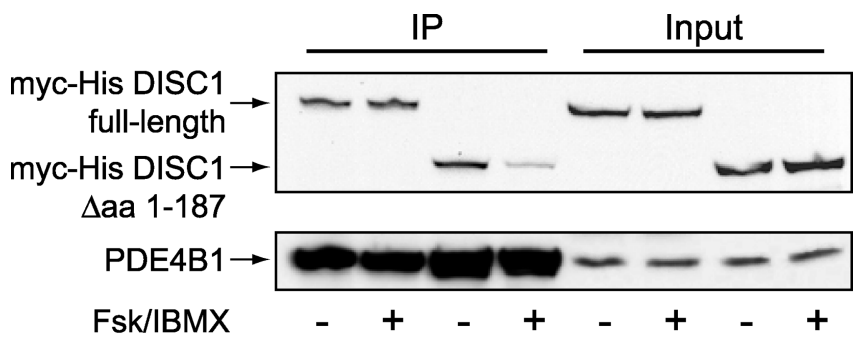

IP:PDE4B

IB:myc

Figure 6. N-terminal truncation of fl-DISC1 sensitizes PDE4B1 to CAMP-mediated release. HEK293 cells cotransfected with PDE4B1 and either myc-HIS-tagged fl-DISC1 or myc-HIStagged DISC1 N-terminal truncate lacking residues 1-187 were treated with vehicle or $100 \mu \mathrm{M}$ forskolin/100 $\mu \mathrm{m} \mathrm{IBMX} \mathrm{for} 15 \mathrm{~min}$ before harvesting and immunoprecipitation with the PDE4B antibody. Coprecipitated myc-HIS-DISC1 species were detected with the anti-myc 9E10 antibody (top). Successful immunocapture of PDE4B1 was demonstrated with the PDE4B antibody (bottom). The input signal shows the expression levels of PDE4B1 (bottom) and myc-HIS DISC1 proteins (top) as determined by direct immunoblotting of $\sim 5 \%$ total cell lysate used for immunoprecipitation assays. IP, Immunoprecipitated; IB, immunoblotted.

not only was still capable of binding to fl-DISC1 but was also released after challenge of cells with forskolin/IBMX (Fig. $8 \mathrm{~A}$ ).

PDE4D3 is unusual among long PDE4 isoforms in that its unique $\mathrm{N}$-terminal region has an additional site for phosphorylation by PKA, namely Ser 13. Phosphorylation at this site has no effect on PKA activity but has been shown to increase the ability of PDE4D3 to bind to mAKAP (Dodge et al., 2001; Carlisle Michel et al., 2004), a PKA scaffolding protein found exclusively in cardiac myocytes and that is upregulated in hypertrophy. A doublemutant construct in which the Ser13 and Ser54 PKA sites in PDE4D3 were ablated by alanine mutation (Ser13Ala:Ser54Ala) had no discernible effect on PDE4D3 interaction with fl-DISC1, and neither did it abrogate the ability of forskolin/IBMX to attenuate the interaction of PDE4D3 with fl-DISC1 (Fig. 8B).

We also generated an N-terminally truncated form of PDE4D3 so as to delete its unique N-terminal region, UCR1 and UCR2, which removes both of the phosphorylation sites for PKA. This construct lacks the UCR2 interaction site for fl-DISC1 but contains the putative fl-DISC1 interaction sites within the CAT unit. Interestingly, this species was both able to coimmunoprecipitate with fl-DISC1 but was also released from DISC1 in cells subject to forskolin/IBMX challenge (Fig. 8C).

\section{Challenge with forskolin/IBMX does not elicit fl-DISC1 phosphorylation}

Although fl-DISC1 is phosphorylated in resting cells where PKA is inactive, there is no increase in fl-DISC1 phosphorylation subsequent to challenge with forskolin/IBMX, and the fl-DISC1 phosphorylation status was unaffected by PKA inhibition with PKI (Fig. 9A). The canonical consensus motif for authentic PKA substrates in vivo is Arg-Arg-X-Ser- $\mathrm{X}_{\text {hydrophobic }}$, although in some instances, weaker substrates are found where Thr substitutes for Ser and Lys substitutes for Arg. The nearest to such a motif in the sequence of fl-DISC1, which can be identified either manually or by Scansite (http://scansite.mit.edu/) analysis, is at Thr744. However, at best, Thr744 might provide a weak putative PKA phosphorylation because it lies within the sequence Lys-Arg-Lys-ThrPro. As a PKA site, it will be compromised by having a side chaindistorting proline next to the target phosphorylation site, a positively charged lysine adjacent to the target phosphorylation site, a Thr rather than a Ser as the target phosphorylation site, and
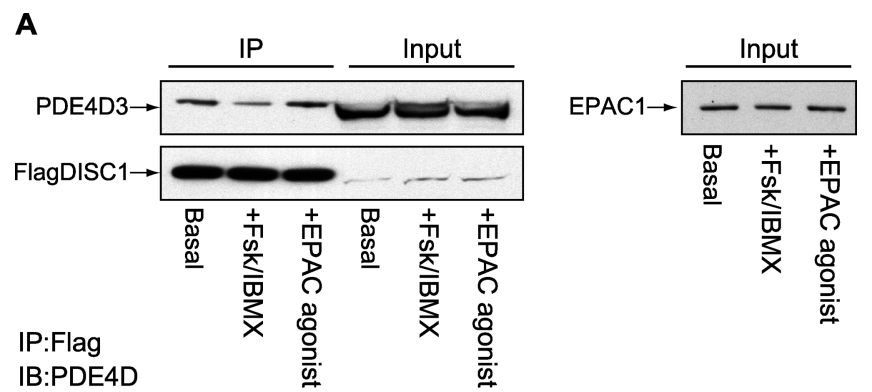

B

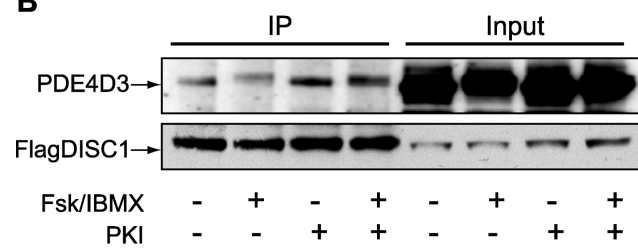

IP:Flag

IB:PDE4D

C

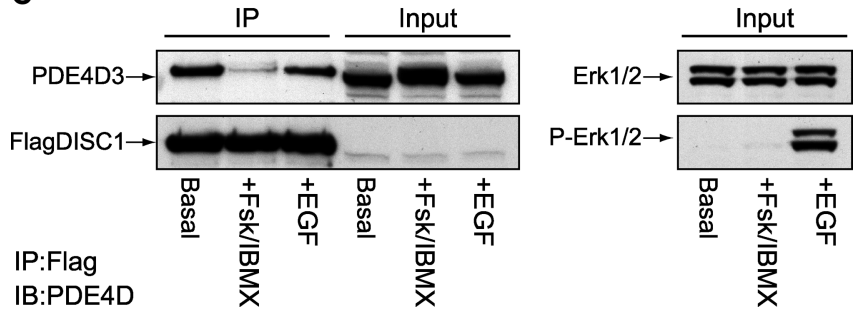

Figure 7. Activation of PKA mediates PDE4D3 release from the fl-DISC1 isoform. A, HEK293 cells exogenously expressing Flag-tagged fI-DISC1, PDE4D3 and EPAC1 were challenged with either vehicle, $100 \mu \mathrm{m}$ forskolin/100 $\mu \mathrm{m}$ IBMX or EPAC agonist (8-pCPT-2'-0-Me-CAMP, $10 \mu \mathrm{m}$ ) for $15 \mathrm{~min}$. FlagDISC1 complexes were isolated from cell lysates by immunoprecipitation with the anti-FLAG M2 antibody, and the PDE4D antibody was used to detect coprecipitated PDE4D3 (top). Equalized capture of FlagDISC1 in immunoprecipitates was determined with the antiFLAG M2 antibody (bottom). The input signals show relative levels of FlagDISC1 (top) and PDE4D3 (bottom) expression in 5\% of the total cell lysate used for immunoprecipitation experiments. Cellular activation of EPAC1 was visualized by immunofluorescent staining of cells with antisera specific for the detection of CAMP-activated EPAC1. Confocal imaging of cells immunostained with the CAMP-activated EPAC1 antibody are shown. B, HEK293 cells were transfected to express either Flag-tagged fl-DISC1, PDE4D3, and empty vector or Flag-tagged $\mathrm{fl}-\mathrm{DISC1}, \mathrm{PDE} 4 \mathrm{D} 3$, and PKI. Cells were then treated with or without $100 \mu \mathrm{m}$ forskolin/100 $\mu \mathrm{M}$ IBMX for 15 min. After harvesting, equal amounts of cell lysate were incubated with the antiFLAG M2 antibody to isolate FlagDISC1 complexes. FlagDISC1 immunoprecipitates were immunoblotted with the PDE4D (top) and anti-FLAG M2 (bottom) antibodies. Relative expression of PDE4D3 and FlagDISC1 in 5\% of cell lysate input for immunoprecipitation experiments is shown in the top and bottom panels, respectively. The cells imaged in $\boldsymbol{A}$, as determined by bright-field microscopy, are shown. C, HEK293 cells coexpressing Flag-tagged fl-DISC1 and PDE4D3 were treated with vehicle, $100 \mu \mathrm{m}$ forskolin/100 $\mu \mathrm{M}$ IBMX, or $200 \mathrm{ng} / \mathrm{ml}$ EGF for 15 min. FlagDISC1 complexes were immunopurified with the anti-FLAG M2 antibody, and the PDE4D antibody was used to detect coprecipitated PDE4D3 (top). Equalized capture of FlagDISC1 in immunoprecipitates was determined with the anti-FLAG M2 antibody (bottom). The input signals show relative levels of FlagDISC1 (top) and PDE4D3 (bottom) expression in $\sim 5 \%$ of the total cell lysate used for immunoprecipitation experiments. EGF-mediated Erk activation was confirmed by immunoblotting of cell lysates with phospho-Erk1/2 antisera (top right). IP, Immunoprecipitated; IB, immunoblotted.

a Lys-Arg rather than Arg-Arg at positions -2 and -3 from the target phosphorylation site. This is consistent, then, with our inability to observe increased phosphorylation of fl-DISC1 in intact cells challenged with forskolin/IBMX (Fig. 9A). 

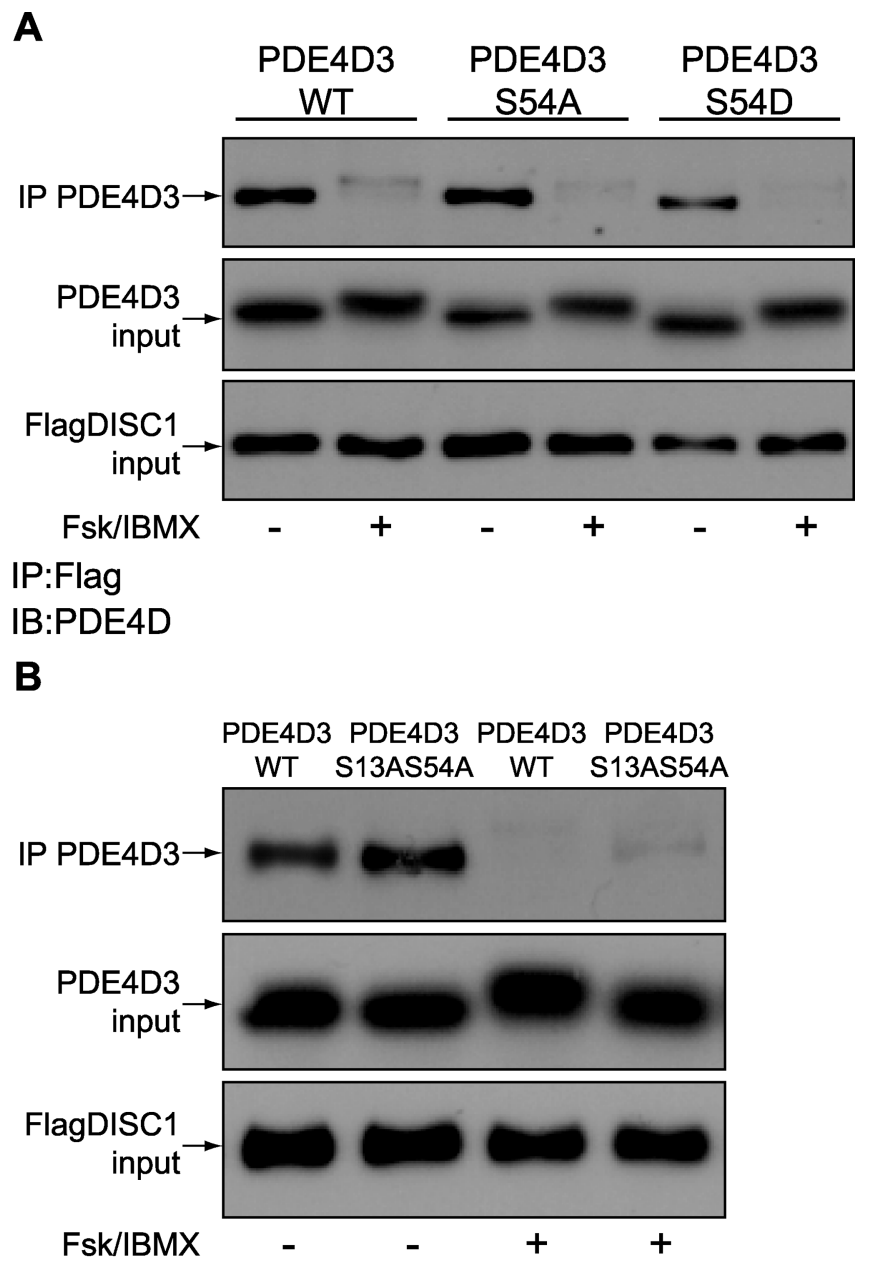

\section{IP:Flag} IB:PDE4D

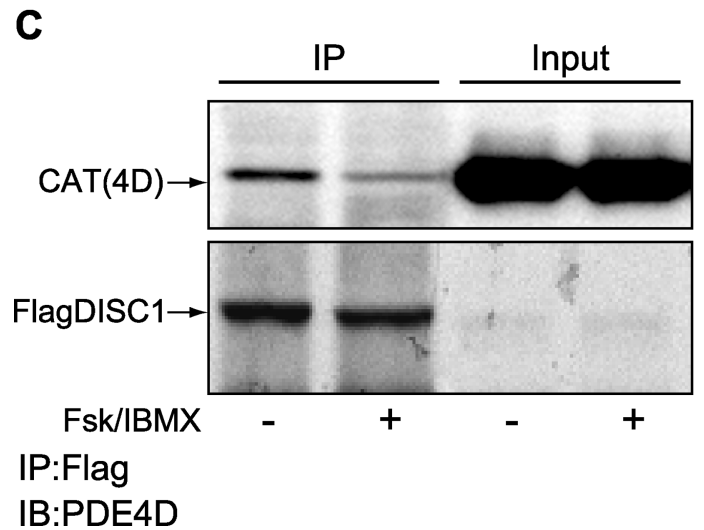

Figure 8. PKA phosphorylation of PDE4D3 is not responsible for PKA-induced dissociation of PDE4D3 from the fl-DISC1 isoform. $A$, HEK293 cells cotransfected with Flag-tagged fl-DISC1 and either wild-type PDE4D3 or PDE4D3 mutants S54A or S54D were challenged with or without 100 $\mu \mathrm{m}$ forskolin/100 $\mu \mathrm{M}$ IBMX. Equal amounts of cell lysate were used to prepare anti-FLAG M2 immunoprecipitates, and coprecipitated PDE4D3 was detected by immunoblotting with the PDE4D antibody (top). Equalized expression of PDE4D3 and FlagDISC1 in input lysates for immunoprecipitation assays was verified by immunoblotting with the PDE4D (middle) and antiFLAG M2 (bottom) antibodies. B, Similar to above, HEK293 cells coexpressing Flag-tagged fl-DISC1 with either wild-type PDE4D3 or PDE4D3 S13A:S54A mutant were treated with or without forskolin/IBMX for $15 \mathrm{~min}$. Cell lysates were then subjected to anti-FLAG M2 immunoprecipitation, and the coprecipitated PDE4D3 was detected with the PDE4D antibody (top). Relative expression inputs of PDE4D3 and FlagDISC1 are shown in the middle and bottom panels, respectively. C, HEK293 cells were cotransfected with Flag-tagged fl-DISC1 and PDE4D CAT domain and treated with vehicle or $100 \mu \mathrm{m}$ forskolin/100 $\mu \mathrm{m}$ IBMX for $15 \mathrm{~min}$. Anti-FLAG
A

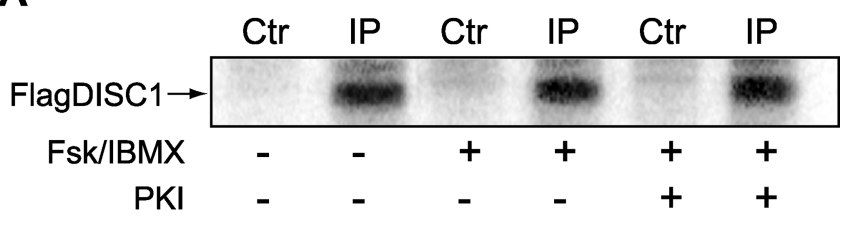

B

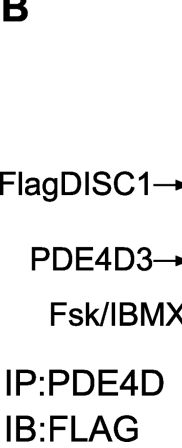

Figure 9. cAMP-induced PKA activation does not elicit PKA phosphorylation of DISC1 in vivo. Attenuation of DISC1:PDE4D3 interaction is not mediated through PKA phosphorylation of DISC1. A, COS1 cells coexpressing Flag-tagged fl-DISC1 plus empty vector or Flag-tagged flDISC1 plus PKI were metabolically labeled with [ ${ }^{32} \mathrm{P}$ ] orthophosphate and challenged with vehicle or $100 \mu \mathrm{m}$ forskolin/100 $\mu \mathrm{m}$ IBMX before harvesting. Equivalent amounts of cell lysate were incubated with anti-FLAG M2 antibody to immunoprecipitate Flag-tagged DISC1. Immunoprecipitates were resolved by SDS-PAGE, and radiolabeled Flag-tagged DISC1 was visualized by autoradiography. $\boldsymbol{B}$, HEK293 cells coexpressing PDE4D3 with either Flag-tagged fl-DISC1 or the Flag-tagged-fl-DISC1 T744A mutant were treated with either vehicle or $100 \mu \mathrm{m}$ forskolin/ $100 \mu \mathrm{M}$ IBMX for $15 \mathrm{~min}$. Equalized cell lysates were incubated with the PDE4D antibody to precipitate PDE4D3, and coprecipitated FlagDISC1 was determined by immunoblotting with the anti-FLAG M2 antibody (top). Immunocapture of PDE4D3 in immunoprecipitates was confirmed with the PDE4D antibody (bottom). Relative expression of Flag-tagged DISC1 and PDE4D3 in 5\% of cell lysate input for immunoprecipitation experiments is shown in the top and bottom panels, respectively. Ctr, Control; IP, immunoprecipitated; IB, immunoblotted.

Nevertheless, to negate any possibility that PKA might phosphorylate fl-DISC1 at Thr744 in these cells, we generated a FLAG-tagged version of fl-DISC1 with a Thr744Ala mutation. Using such a construct, we were able to find that not only could it coimmunoprecipitate with PDE4D3 but that PDE4D3 was released from such a mutant form of fl-DISC1 when transfected cells were challenged with forskolin/IBMX (Fig. 9B).

\section{Discussion}

cAMP and the control of its degradation by PDEs, including PDE4, has a well appreciated role in regulating learning and memory processes (Barad et al., 1998; O'Donnell and Zhang, 2004; Arnsten et al., 2005; Blokland et al., 2006). Recently, we have shown that DISC1 interacts with PDE4B (Millar et al., 2005; Cheung et al., 2007). This is likely to be of importance because PDE4 recruitment to signaling protein complexes confers spatial and temporal regulation of localized cAMP levels (Baillie et al., 2005). fl-DISC1 interacts with members from all four PDE4 subfamilies (Fig. 1), implying the presence of a conserved binding site(s). However, profound functional differences between PDE4

$\leftarrow$

M2 antibody was used to immunoprecipitate FlagDISC1, and coprecipitating CAT was visualized by immunoblotting with the PDE4D antibody (top). Equalized expression of CAT and FlagDISC1 in input lysates for immunoprecipitation assays was verified by immunoblotting with the PDE4D (top) and anti-FLAG M2 (bottom) antibodies. IP, Immunoprecipitated; IB, immunoblotted; WT, wild type. 
subfamilies were evident because elevating intracellular cAMP levels caused release of PDE4C and PDE4D but not PDE4A and PDE4B (Figs. 1, 2). This contrasts with previous studies identifying PDE4B as a novel binding partner for the $71 \mathrm{kDa}$ DISC1 isoform in SHSY5Y neuroblastoma cells that is released when intracellular cAMP levels increase (Millar et al., 2005). In contrast to fl-DISC1, the exact nature of the short $71 \mathrm{kDa}$ DISC1 isoform is unknown. Thus, DISC1-PDE4 interactions are more complex than previously envisaged, with factors such as DISC1 and PDE4 isoform expression being defining criteria.

To gain insight into fl-DISC1/PDE4 binding regions, we used a novel peptide array strategy, which we have used to identify PDE4 binding sites on the scaffold proteins $\beta$ arrestin, RACK1, and AKAP18 (Bolger et al., 2006; Baillie et al., 2007; Stefan et al., 2007). Here we focused on PDE4B and PDE4D as examples of subfamilies that bind fl-DISC1 but differ in their sensitivity to dissociation by elevated intracellular cAMP. Using fl-DISC1 arrays, we identified two binding sites common to PDE4B and PDE4D, one (cs1) located between amino acids 191-230 of flDISC1 and one (cs2) located within a predicted C-terminal coiled-coil domain (amino acids 611-650) (Fig. 3C). Indeed, various proteins that sequester PDE4 use multiple binding sites (MacKenzie et al., 2000; Bolger et al., 2006; Baillie et al., 2007). $\mathrm{N}$-terminal truncation, leading to removal of cs1 as well as PDE4B-specific sites, abrogated PDE4B1 interaction with flDISC1 (Millar et al., 2005), implying that multiple sites are required for complex stabilization in vivo.

UCR2, which is conserved through PDE4 subfamilies, was suggested as interacting with fl-DISC1 (Millar et al., 2005), implying that UCR2 binds a common site on fl-DISC1. Consistent with this, probing fl-DISC1 arrays with UCR2 (Fig. 3H) demonstrated interaction with cs1 only (Fig. 3C).

The cs2 site in fl-DISC1 (Fig. $3 C$ ) should interact with a conserved region of PDE4. Indeed, probing fl-DISC1 arrays with the PDE4D CAT unit identified the cs2 site (Fig. $3 H$ ). Surprisingly, this probe also identified cs1 (Fig. $3 H$ ). However, both cs1 and cs2 share a motif (vide supra) that may allow the PDE4 CAT unit to bind each region in vitro. Indeed, the presence of both cs 1 and cs2 in fl-DISC1 is likely to ensure fidelity of UCR2 association with cs1 and of the PDE4 CAT unit with cs2, because this will maximize PDE4/fl-DISC1 contact.

While probing fl-DISC1 arrays with PDE4D3 identified only cs1 and cs2 sites, probing with PDE4B1 identified additional binding sites located within the fl-DISC1 N-terminal region (Fig. 3) presumed as either missing or altered in short DISC1 isoforms. Such PDE4B-specific binding sites on fl-DISC1 may protect the complex from dissociation when intracellular cAMP levels are elevated.

Consistent with the above analyses, performing reciprocal studies, when PDE4B and PDE4D peptide arrays were probed with fl-DISC1, we identified two common sites of interaction: one within UCR2 (Fig. 4C) and the other within a highly conserved region of the CAT unit (Fig. $4 F$ ).

Array analyses also indicated a site for fl-DISC1 interaction that was unique to PDE4B and located within a partially conserved region of the CAT unit (Fig. $5 A-C$ ). This may interact with the PDE4B-specific binding sites on fl-DISC1. We also identified a putative binding site for fl-DISC1 unique to PDE4D and located within a region of its CAT unit that has significant sequence disparity to the equivalent PDE4B region (Fig. 5F). However, PDE4D appears to interact only with the cs1 and cs2 sites in the fl-DISC1 peptide array. Thus, either folded fl-DISC1 is required to interact with this PDE4D specific binding site or, while exposed in the peptide array, it remains cryptic in the folded
PDE4D. Notwithstanding this, we show here that cell-permeable peptides reflecting cs1 and cs2 of DISC1 suffice to disrupt interaction of fl-DISC1 in cells with PDE4D3, but not PDE4B1 (Fig. $3 I, J)$. This supports the contention that fl-DISC1 sequesters PDE4D3 through cs1/cs2 and that PDE4B1 interaction involves additional sites (Fig. 3C).

Disruption of fl-DISC1-PDE4D3 complexes occurs during PKA activation (Figs. 1, 2). This is not attributable to PKA phosphorylation of PDE4D3 because complex dissociation during PKA activation was not ablated after Ala mutagenesis (Fig. 8) of either of the two PKA sites in PDE4D3, Ser54 or Ser13 (Sette and Conti, 1996; MacKenzie et al., 2002). fl-DISC1 is a phosphoprotein (Fig. 9A), although the identity of the phosphorylation site(s) is unknown. Basal phosphorylation is not attributable to PKA because it was unaffected by expression of the PKA inhibitor PKI (Fig. 9A). Furthermore, fl-DISC1 phosphorylation was not increased after forskolin/IBMX challenge, which activates PKA in these cells (Lynch et al., 2005; Baillie et al., 2007). Indeed, sequence analysis reveals that there is no obvious PKA phosphorylation consensus motif. The closest sequence that can be identified, either manually or by Scansite (http://scansite.mit.edu/) analysis, is at Thr744. This, at best, might provide a weak site for PKA phosphorylation, lying within the sequence Lys-Arg-LysThr-Pro. To negate any possible phosphorylation of Thr744, we mutated it to alanine in fl-DISC1. Such mutation failed to either alter PDE4D3-fl-DISC1 complex formation or prevent disruption in cells challenged with forskolin/IBMX (Fig. 9B).

Binding at the PDE4B-specific sites on fl-DISC1 likely protects PDE4B from cAMP/PKA-mediated release because their removal, by $\mathrm{N}$-terminal deletion, generated a DISC1 truncate that released PDE4B1 after elevation of intracellular cAMP (Fig. 6). This species may mimic the short $71 \mathrm{kDa}$ DISC1 isoform, which is the major DISC1 species expressed endogenously in SHSY5Y cells (Millar et al., 2005). Although the sequence of the $71 \mathrm{kDa}$ DISC1 isoform remains to be elucidated, it is believed to be N-terminally truncated compared with fl-DISC1 (Millar et al., 2004), which would be consistent with our studies.

What, then, is the PKA-mediated process that destabilizes PDE4D3-fl-DISC1 complexes? One possibility is that PKA phosphorylation of a protein may either increase its affinity for binding PDE4D or generate an ability to bind PDE4D. Another is that PKA phosphorylation of a protein alters its interaction with flDISC1, thereby attenuating PDE4D3 binding. The resolution of this will require a major proteomics investigation that is beyond the remit of this study. However, the principle that PDE4 isoforms can redistribute dynamically between signaling scaffolds has been firmly established for PDE4D5 sequestered to RACK1 and $\beta$ arrestin scaffolds (Lynch et al., 2005; Bolger et al., 2006).

This study reveals the complex nature of DISC1-PDE4 interactions. The diversity of PDE4 subfamilies and DISC1 isoforms has functional correlates exemplified in the different susceptibilities of partnerships to be dissociated after cAMP elevation. flDISC1 comprises a globular N-terminal head domain with a C-terminal tail containing a series of coiled-coil domains (Millar et al., 2000) (Fig. 3C). The head domain contains four of the five putative PDE4B-specific contact sites (Fig. 3C), suggesting that $\mathrm{PDE} 4 \mathrm{~B}$ binding is likely a major function of this region. Both cs1 and cs2 are located within regions of DISC1 that are well conserved between species, consistent with their importance for PDE4 interaction. Furthermore, exon 9, which encodes cs2, may be alternatively spliced such that cs2 is absent from certain DISC1 transcripts (Ma et al., 2002; Millar et al., 2004), thereby compromising PDE4 interaction. Alterations in DISC1 and PDE4 iso- 
form expression profiles therefore offer a means to reprogram whether these proteins interact or DISC1-sequestered PDE4 is released after cAMP elevation. Thus functional variation, genetically determined, epigenetically programmed, or environmentally cued, that influences isoform expression or binding affinity within individual contact sites may be of importance in mental illness. Although genetic studies have identified DISC1, PDE4B, and $P D E 4 D$ as risk factors for major mental illness, no definitive causal variants, beyond chromosomal translocations (Millar et al., 2005), have been identified in psychiatric patients. However two point mutations in the mouse Discl gene confer behavioral, brain structural, and pharmacological phenotypes related to schizophrenia and depression (Clapcote et al., 2007). These mutations are located within regions of mouse Discl corresponding to the PDE4B-specific binding sites $4 \mathrm{bss} 1$ and $4 \mathrm{bss} 2$ of human DISC1 (Fig. 3C) and both reduce Disc1 binding to PDE4B (Clapcote et al., 2007). We show here that peptides encompassing these regions bind PDE4B1 and substitutions (Ala/Pro) at Leu100, which reflect the consequences of point mutations, attenuate PDE4B1 interaction (Fig. $3 F, G$ ). Thus, genetic variation in either DISC1 or PDE4 that alters their coexpression, dynamic association, and thereby cAMP signaling may be predicted to predispose to psychopathology. Such aberrant interactions are a potential target for novel drug and diagnostic development.

\section{References}

Arnsten AF, Ramos BP, Birnbaum SG, Taylor JR (2005) Protein kinase A as a therapeutic target for memory disorders: rationale and challenges. Trends Mol Med 11:121-128.

Baillie GS, Huston E, Scotland G, Hodgkin M, Gall I, Peden AH, MacKenzie C, Houslay ES, Currie R, Pettitt TR, Walmsley AR, Wakelam MJ, Warwicker J, Houslay MD (2002) TAPAS-1, a novel microdomain within the unique $\mathrm{N}$-terminal region of the PDE4A1 cAMP-specific phosphodiesterase that allows rapid, Ca2 +-triggered membrane association with selectivity for interaction with phosphatidic acid. J Biol Chem 277:28298-28309.

Baillie GS, Scott JD, Houslay MD (2005) Compartmentalisation of phosphodiesterases and protein kinase A: opposites attract. FEBS Lett 579:3264-3270.

Baillie GS, Adams DR, Bahri N, Houslay TM, Vadrevu S, Meng D, Li X, Dunlop A, Milligan G, Bolger GB, Klussmann E, Houslay MD (2007) Mapping binding sites for the PDE4D5 cAMP-specific phosphodiesterase to the N-and C-domains of betaarrestin using spot-immobilised peptide arrays. Biochem J 404:71-80.

Barad M, Bourtchouladze R, Winder DG, Golan H, Kandel E (1998) Rolipram, a type IV-specific phosphodiesterase inhibitor, facilitates the establishment of long-lasting long-term potentiation and improves memory. Proc Natl Acad Sci USA 95:15020-15025.

Bardwell L, Cooper AJ, Friedberg EC (1992) Stable and specific association between the yeast recombination and DNA repair proteins RAD1 and RAD10 in vitro. Mol Cell Biol 12:3041-3049.

Beard MB, Olsen AE, Jones RE, Erdogan S, Houslay MD, Bolger GB (2000) UCR1 and UCR2 domains unique to the cAMP-specific phosphodiesterase family form a discrete module via electrostatic interactions. J Biol Chem 275:10349-10358.

Blokland A, Schreiber R, Prickaerts J (2006) Improving memory: a role for phosphodiesterases. Curr Pharm Des 12:2511-2523.

Bolger GB, Erdogan S, Jones RE, Loughney K, Scotland G, Hoffmann R, Wilkinson I, Farrell C, Houslay MD (1997) Characterization of five different proteins produced by alternatively spliced mRNAs from the human cAMP-specific phosphodiesterase PDE4D gene. Biochem J 328:539-548.

Bolger GB, Baillie GS, Li X, Lynch MJ, Herzyk P, Mohamed A, Mitchell LH, McCahill A, Hundsrucker C, Klussmann E, Adams DR, Houslay MD (2006) Scanning peptide array analyses identify overlapping binding sites for the signalling scaffold proteins, beta-arrestin and RACK1, in cAMP-specific phosphodiesterase PDE4D5. Biochem J 398:23-36.

Bos JL (2006) Epac proteins: multi-purpose cAMP targets. Trends Biochem Sci 31:680-686.

Brandon NJ, Handford EJ, Schurov I, Rain JC, Pelling M, Duran-Jimeniz B, Camargo LM, Oliver KR, Beher D, Shearman MS, Whiting PJ (2004)
Disrupted in schizophrenia 1 and Nudel form a neurodevelopmentally regulated protein complex: implications for schizophrenia and other major neurological disorders. Mol Cell Neurosci 25:42-55.

Camargo LM, Collura V, Rain JC, Mizuguchi K, Hermjakob H, Kerrien S, Bonnert TP, Whiting PJ, Brandon NJ (2007) Disrupted in schizophrenia 1 interactome: evidence for the close connectivity of risk genes and a potential synaptic basis for schizophrenia. Mol Psychiatry 12:74-86.

Carlisle Michel JJ, Dodge KL, Wong W, Mayer NC, Langeberg LK, Scott JD (2004) PKA-phosphorylation of PDE4D3 facilitates recruitment of the mAKAP signalling complex. Biochem J 381:587-592.

Cheung YF, Kan Z, Garrett-Engele P, Gall I, Murdoch H, Baillie GS, Camargo LM, Johnson JM, Houslay MD, Castle JC (2007) PDE4B5, a novel, super-short, brain-specific cAMP phosphodiesterase-4 variant whose isoform-specifying $\mathrm{N}$-terminal region is identical to that of cAMP phosphodiesterase-4D6 (PDE4D6). J Pharmacol Exp Ther 332:600-609.

Clapcote SJ, Lipina TV, Millar JK, Mackie S, Christie S, Ogawa F, Lerch JP, Trimble K, Uchiyama M, Sakuraba Y, Kaneda H, Shiroishi T, Houslay MD, Henkelman RM, Sled JG, Gondo Y, Porteous DJ, Roder JC (2007) Behavioral phenotypes of Discl missense mutations in mice. Neuron 54:387-402.

Conti M, Richter W, Mehats C, Livera G, Park JY, Jin C (2003) Cyclic AMPspecific PDE4 phosphodiesterases as critical components of cyclic AMP signaling. J Biol Chem 278:5493-5496.

Davis RL, Cherry J, Dauwalder B, Han PL, Skoulakis E (1995) The cyclic AMP system and Drosophila learning. Mol Cell Biochem 149-150:271-278.

Dodge KL, Khouangsathiene S, Kapiloff MS, Mouton R, Hill EV, Houslay MD, Langeberg LK, Scott JD (2001) mAKAP assembles a protein kinase A/PDE4 phosphodiesterase cAMP signaling module. EMBO J 20:1921-1930.

Engels P, Abdel'Al S, Hulley P, Lubbert H (1995) Brain distribution of four rat homologues of the Drosophila dunce cAMP phosphodiesterase. J Neurosci Res 41:169-178.

Fleming YM, Frame MC, Houslay MD (2004) PDE4-regulated cAMP degradation controls the assembly of integrin-dependent actin adhesion structures and REF52 cell migration. J Cell Sci 117:2377-2388.

Gao Y, Nikulina E, Mellado W, Filbin MT (2003) Neurotrophins elevate cAMP to reach a threshold required to overcome inhibition by MAG through extracellular signal-regulated kinase-dependent inhibition of phosphodiesterase. J Neurosci 23:11770-11777.

Hashimoto R, Numakawa T, Ohnishi T, Kumamaru E, Yagasaki Y, Ishimoto T, Mori T, Nemoto K, Adachi N, Izumi A, Chiba S, Noguchi H, Suzuki T, Iwata N, Ozaki N, Taguchi T, Kamiya A, Kosuga A, Tatsumi M, Kamijima K, et al. (2006) Impact of the DISC1 Ser704Cys polymorphism on risk for major depression, brain morphology and ERK signaling. Hum Mol Genet 15:3024-3033.

Hoffmann R, Wilkinson IR, McCallum JF, Engels P, Houslay MD (1998) cAMP-specific phosphodiesterase HSPDE4D3 mutants which mimic activation and changes in rolipram inhibition triggered by protein kinase $\mathrm{A}$ phosphorylation of Ser-54: generation of a molecular model. Biochemical J 333:139-149.

Houslay MD, Adams DR (2003) PDE4 cAMP phosphodiesterases: modular enzymes that orchestrate signalling cross-talk, desensitization and compartmentalization. Biochem J 370:1-18.

Houslay MD, Schafer P, Zhang KY (2005) Keynote review: phosphodiesterase-4 as a therapeutic target. Drug Discov Today 10:1503-1519.

Hundsrucker C, Krause G, Beyermann M, Prinz A, Zimmermann B, Diekmann O, Lorenz D, Stefan E, Nedvetsky P, Dathe M, Christian F, McSorley T, Krause E, McConnachie G, Herberg FW, Scott JD, Rosenthal W, Klussmann E (2006) High-affinity AKAP7delta-protein kinase A interaction yields novel protein kinase A-anchoring disruptor peptides. Biochem J 396:297-306.

Huston E, Pooley L, Julien P, Scotland G, McPhee I, Sullivan M, Bolger G, Houslay MD (1996) The human cyclic AMP-specific phosphodiesterase PDE-46 (HSPDE4A4B) expressed in transfected COS7 cells occurs as both particulate and cytosolic species that exhibit distinct kinetics of inhibition by the antidepressant rolipram. J Biol Chem 271:31334-31344.

Huston E, Lumb S, Russell A, Catterall C, Ross AH, Steele MR, Bolger GB, Perry MJ, Owens RJ, Houslay MD (1997) Molecular cloning and transient expression in COS7 cells of a novel human PDE4B cAMP-specific phosphodiesterase, HSPDE4B3. Biochem J 328:549-558.

James R, Adams RR, Christie S, Buchanan SR, Porteous DJ, Millar JK (2004) Disrupted in schizophrenia 1 (DISC1) is a multicompartmentalized protein that predominantly localizes to mitochondria. Mol Cell Neurosci 26:112-122. 
Kamiya A, Kubo K, Tomoda T, Takaki M, Youn R, Ozeki Y, Sawamura N, Park U, Kudo C, Okawa M, Ross CA, Hatten ME, Nakajima K, Sawa A (2005) A schizophrenia-associated mutation of DISC1 perturbs cerebral cortex development. Nat Cell Biol 7:1167-1178.

Kyosseva SV (2004) The role of the extracellular signal-regulated kinase pathway in cerebellar abnormalities in schizophrenia. Cerebellum 3:94-99.

Laemmli UK (1970) Cleavage of structural proteins during the assembly of the head of bacteriophage T4. Nature 227:680-685.

Liu YL, Fann CS, Liu CM, Chen WJ, Wu JY, Hung SI, Chen CH, Jou YS, Liu SK, Hwang TJ, Hsieh MH, Ouyang WC, Chan HY, Chen JJ, Yang WC, Lin CY, Lee SF, Hwu HG (2006) A single nucleotide polymorphism fine mapping study of chromosome 1q42.1 reveals the vulnerability genes for schizophrenia, GNPAT and DISC1: association with impairment of sustained attention. Biol Psychiatry 60:554-562.

Lynch MJ, Baillie GS, Mohamed A, Li X, Maisonneuve C, Klussmann E, van Heeke G, Houslay MD (2005) RNA silencing identifies PDE4D5 as the functionally relevant cAMP phosphodiesterase interacting with beta arrestin to control the protein kinase A/AKAP79-mediated switching of the beta2-adrenergic receptor to activation of ERK in HEK293B2 cells. J Biol Chem 280:33178-33189.

Ma L, Liu Y, Ky B, Shughrue PJ, Austin CP, Morris JA (2002) Cloning and characterization of Disc1, the mouse ortholog of DISC1 (disrupted-inschizophrenia 1). Genomics 80:662-672.

MacKenzie SJ, Houslay MD (2000) Action of rolipram on specific PDE4 cAMP phosphodiesterase isoforms and on the phosphorylation of CAMPresponse-element-binding protein $(\mathrm{CREB})$ and p38 mitogen-activated protein (MAP) kinase in U937 monocytic cells. Biochem J 347:571-578.

MacKenzie SJ, Baillie GS, McPhee I, Bolger GB, Houslay MD (2000) ERK2 MAP kinase binding, phosphorylation and regulation of PDE4D cAMP specific phosphodiesterases: the involvement of C-terminal docking sites and N-terminal UCR regions. J Biol Chem 275:16609-16617.

MacKenzie SJ, Baillie GS, McPhee I, MacKenzie C, Seamons R, McSorley T, Millen J, Beard MB, van Heeke G, Houslay MD (2002) Long PDE4 cAMP specific phosphodiesterases are activated by protein kinase A-mediated phosphorylation of a single serine residue in upstream conserved region 1 (UCR1). Br J Pharmacol 136:421-433.

Millar JK, Wilson-Annan JC, Anderson S, Christie S, Taylor MS, Semple CA, Devon RS, Clair DM, Muir WJ, Blackwood DH, Porteous DJ (2000) Disruption of two novel genes by a translocation co-segregating with schizophrenia. Hum Mol Genet 9:1415-1423.

Millar JK, James R, Brandon NJ, Thomson PA (2004) DISC1 and DISC2: discovering and dissecting molecular mechanisms underlying psychiatric illness. Ann Med 36:367-378.

Millar JK, Pickard BS, Mackie S, James R, Christie S, Buchanan SR, Malloy MP, Chubb JE, Huston E, Baillie GS, Thomson PA, Hill EV, Brandon NJ, Rain JC, Camargo LM, Whiting PJ, Houslay MD, Blackwood DH, Muir WJ, Porteous DJ (2005) DISC1 and PDE4B are interacting genetic factors in schizophrenia that regulate cAMP signaling. Science 310:1187-1191.

Miro X, Perez-Torres S, Puigdomenech P, Palacios JM, Mengod G (2002) Differential distribution of PDE4D splice variant mRNAs in rat brain suggests association with specific pathways and presynaptical localization. Synapse 45:259-269.

Miyoshi K, Asanuma M, Miyazaki I, Diaz-Corrales FJ, Katayama T, Tohyama M, Ogawa N (2004) DISC1 localizes to the centrosome by binding to kendrin. Biochem Biophys Res Commun 317:1195-1199.

Morris JA, Kandpal G, Ma L, Austin CP (2003) DISC1 (disrupted-inschizophrenia 1) is a centrosome-associated protein that interacts with MAP1A, MIPT3, ATF4/5 and NUDEL: regulation and loss of interaction with mutation. Hum Mol Genet 12:1591-1608.

Nikulina E, Tidwell JL, Dai HN, Bregman BS, Filbin MT (2004) The phosphodiesterase inhibitor rolipram delivered after a spinal cord lesion promotes axonal regeneration and functional recovery. Proc Natl Acad Sci USA 101:8786-8790.

O’Donnell JM, Zhang HT (2004) Antidepressant effects of inhibitors of cAMP phosphodiesterase (PDE4). Trends Pharmacol Sci 25:158-163.

Ogawa F, Kasai M, Akiyama T (2005) A functional link between disruptedin-schizophrenia 1 and the eukaryotic translation initiation factor 3. Biochem Biophys Res Commun 338:771-776.

Owens RJ, Lumb S, Rees-Milton K, Russell A, Baldock D, Lang V, Crabbe T, Ballesteros M, Perry MJ (1997) Molecular cloning and expression of a human phosphodiesterase 4C. Cell Signal 9:575-585.

Ozeki Y, Tomoda T, Kleiderlein J, Kamiya A, Bord L, Fujii K, Okawa M,
Yamada N, Hatten ME, Snyder SH, Ross CA, Sawa A (2003) Disruptedin-schizophrenia-1 (DISC-1): mutant truncation prevents binding to NudE-like (NUDEL) and inhibits neurite outgrowth. Proc Natl Acad Sci USA 100:289-294.

Pearse DD, Pereira FC, Marcillo AE, Bates ML, Berrocal YA, Filbin MT, Bunge MB (2004) cAMP and Schwann cells promote axonal growth and functional recovery after spinal cord injury. Nat Med 10:610-616.

Pickard BS, Thomson PA, Christoforou A, Evans KL, Morris SW, Porteous DJ, Blackwood DHR, Muir WJ (2007) The PDE4B gene confers sexspecific protection against schizophrenia. Psychiatr Genet 17:129-133.

Polesskaya OO, Smith RF, Fryxell KJ (2007) Chronic nicotine doses downregulate PDE4 isoforms that are targets of antidepressants in adolescent female rats. Biol Psychiatry 61:56-64.

Porteous DJ, Millar JK (2006) Disrupted in schizophrenia 1: building brains and memories. Trends Mol Med 12:255-261.

Porteous DJ, Thomson P, Brandon NJ, Millar JK (2006) The genetics and biology of DISC1-an emerging role in psychosis and cognition. Biol Psychiatry 60:123-131.

Qu M, Tang F, Yue W, Ruan Y, Lu T, Liu Z, Zhang H, Han Y, Zhang D, Wang F, Zhang D (2007) Positive association of the disrupted-inschizophrenia-1 gene (DISC1) with schizophrenia in the Chinese han population. Am J Med Genet B Neuropsychiatr Genet 144:266-270.

Richter W, Jin SL, Conti M (2005) Splice variants of the cyclic nucleotide phosphodiesterase PDE4D are differentially expressed and regulated in rat tissue. Biochem J 388:803-811.

Sette C, Conti M (1996) Phosphorylation and activation of a cAMP-specific phosphodiesterase by the CAMP-dependent protein kinase. Involvement of serine 54 in the enzyme activation. J Biol Chem 271:16526-16534.

Sette C, Iona S, Conti M (1994) The short-term activation of a rolipramsensitive, cAMP-specific phosphodiesterase by thyroid-stimulating hormone in thyroid FRTL-5 cells is mediated by a cAMP-dependent phosphorylation. J Biol Chem 269:9245-9252.

Shinoda T, Taya S, Tsuboi D, Hikita T, Matsuzawa R, Kuroda S, Iwamatsu A, Kaibuchi K (2007) DISC1 regulates neurotrophin-induced axon elongation via interaction with Grb2. J Neurosci 27:4-14.

Smith KJ, Scotland G, Beattie J, Trayer IP, Houslay MD (1996) Determination of the structure of the N-terminal splice region of the cyclic AMPspecific phosphodiesterase RD1 (RNPDE4A1) by 1H NMR and identification of the membrane association domain using chimeric constructs. J Biol Chem 271:16703-16711.

Stefan E, Wiesner B, Baillie GS, Mollajew R, Henn V, Lorenz D, Furkert J, Santamaria K, Nedvetsky P, Hundsrucker C, Beyermann M, Krause E, Pohl P, Gall I, MacIntyre AN, Bachmann S, Houslay MD, Rosenthal W, Klussmann E (2007) Compartmentalization of cAMP-dependent signaling by phosphodiesterase-4D is involved in the regulation of vasopressin-mediated water reabsorption in renal principal cells. J Am Soc Nephrol 18:199-212.

Sweatt JD (2004) Mitogen-activated protein kinases in synaptic plasticity and memory. Curr Opin Neurobiol 14:311-317.

Tasken K, Aandahl EM (2004) Localized effects of cAMP mediated by distinct routes of protein kinase A. Physiol Rev 84:137-167.

Taya S, Shinoda T, Tsuboi D, Asaki J, Nagai K, Hikita T, Kuroda S, Kuroda K, Shimizu M, Hirotsune S, Iwamatsu A, Kaibuchi K (2007) DISC1 regulates the transport of the NUDEL/LIS1/14-3-3e complex through kinesin-1. J Neurosci 27:15-26.

Thompson BE, Freking F, Pho V, Schlinger BA, Cherry JA (2000) Cyclic AMP phosphodiesterases in the zebra finch: distribution, cloning and characterization of a PDE4B homolog. Brain Res Mol Brain Res 83:94-106.

Tomppo L, Hennah W, Lahermo P, Loukola A, Ekelund J, Partonen T, Palotie A, Lonnqvist J, Peltonen L (2006) Association evidence from NUDEL and PDE4D support the DISC1-pathway concept in the etiology of schizophrenia. Am J Med Genet B Neuropsychiatr Genet 141B:717.

Zhang HT, Huang Y, Suvarna NU, Deng C, Crissman AM, Hopper AT, De Vivo M, Rose GM, O'Donnell JM (2005) Effects of the novel PDE4 inhibitors MEM1018 and MEM1091 on memory in the radial-arm maze and inhibitory avoidance tests in rats. Psychopharmacology (Berl) 179:613-619.

Zhang HT, Zhao Y, Huang Y, Deng C, Hopper AT, De Vivo M, Rose GM, O’Donnell JM (2006) Antidepressant-like effects of PDE4 inhibitors mediated by the high-affinity rolipram binding state (HARBS) of the phosphodiesterase-4 enzyme (PDE4) in rats. Psychopharmacology (Berl) 186:209-217. 